Unfallchirurg 2020 · 123:309-325

https://doi.org/10.1007/s00113-020-00793-8

Online publiziert: 11. März 2020

(c) Der/die Autor(en) 2020

Wissenschaftliche Leitung

P. Biberthaler, München

T. Gösling, Braunschweig

T. Mittlmeier, Rostock
CME

Zertifizierte Fortbildung

\section{Suprakondyläre Humerusfraktur im Kindesalter}

Online teilnehmen unter: www.springermedizin.de/cme

Für diese Fortbildungseinheit werden 3 Punkte vergeben.

\section{Kontakt}

Springer Medizin Kundenservice Tel. 08007780777

(kostenfrei in Deutschland)

E-Mail:

kundenservice@springermedizin.de

\section{Informationen}

zur Teilnahme und Zertifizierung finden Sie im CME-Fragebogen am Ende des Beitrags.

\author{
Annelie-Martina Weinberg ${ }^{1,2} \cdot$ Benjamin Frei ${ }^{3} \cdot$ Patrik Holweg \\ ${ }^{1}$ Univ. Klinik für Orthopädie und Orthopädische Chirurgie, Medizinische Universität Graz, Graz, Österreich \\ ${ }^{2}$ Orthopädie \& Traumatologie, Landesklinikum Baden-Mödling, Standort Mödling, Mödling, Österreich \\ ${ }^{3}$ Abteilung für Kinderchirurgie, Universitäts-Kinderspital beider Basel (UKBB), Basel, Schweiz
}

\title{
Zusammenfassung
}

Die Einteilung der suprakondylären Oberarmfraktur erfolgt im deutschsprachigen Raum nach der Von-Laer-Klassifikation, die von der AO übernommen wurde und den Vorteil bietet, dass daraus die Therapie ableitbar ist. Wenn indiziert, wird die sofortige Operation einer zeitnahen verzögerten Versorgung vorgezogen. Das Ergebnis wird anhand funktioneller Tests direkt im OP kontrolliert. Frakturinstabilität und korrektes Platzieren der Kirschner(K)-Drähte stellen Herausforderungen dar. Alternativen sind Fixateur externe und elastisch stabile intramedulläre Nagelung (ESIN). Begleitende Verletzungen betreffen initial den N. medianus und die A. brachialis, in zweiter Linie den N. radialis. Der N.-ulnaris-Schaden ist meist eine postoperative Komplikation. Die knöcherne Konsolidation ist nach 3 bis 4 Wochen gegeben; danach können eingebrachte Implantate entfernt werden. Versenkte K-Drähte und ESIN werden nach 3 bis 6 Monaten entfernt, je nach operativer Kapazität und Beschwerden des Patienten.

\section{Schlüsselwörter}

Klassifikation · Konservative Behandlung · Frakturfixation · Knochenfehlstellung · Nachbehandlung

\section{Lernziele}

Nach Lektüre dieses Beitrags können Sie ...

- zuverlässig die suprakondylären Oberarmfrakturen im Kindesalter einteilen.

- die Problematik der Fraktur verstehen.

- einen Therapiealgorithmus anwenden.

- Komplikationen der Fraktur kennen.

- verschiedene operative und konservative Versorgungstechniken anwenden. 


\section{Einleitung}

Die suprakondyläre Humerusfraktur ist die Fraktur im Kindesalter, die nach wie vor die am meisten untersuchte und in PubMed publizierte Entität ist (Tab. 1). Damit stellt sich die Frage nach dem Warum. Ist es die Fraktur, die die meisten Probleme in der Anwendung des korrekten Therapiealgorithmus darstellt, oder die operative Versorgung, die insgesamt immer noch unbefriedigende Ergebnisse nach sich zieht?

\section{Epidemiologie}

Die suprakondyläre Oberarmfraktur ist definitionsgemäß eine distale metaphysäre Oberarmfraktur und keine Gelenkfraktur. Sie findet sich innerhalb der kindlichen Gelenkskapsel. Sie weist somit keine intraartikulären Frakturanteile auf. Sie zieht aber nach einer Fehlheilung eine störende kosmetische Deformität des Ellenbogens und nur in seltenen Fällen eine funktionelle Störung nach sich [1].

\section{- Merke}

Die suprakondyläre Oberarmfraktur ist keine Gelenkfraktur, sondern eine distale metaphysäre Fraktur des Humerusschafts.

Diese Frakturen kommen spezifisch im Kindesalter um das 4. bis 7. Lebensjahr vor. Dies ist auf die Umformung des distalen Humerus während des Wachstums des Kindes und des Ellenbogens zurückzuführen [1, 2, 3]. Es zeigt sich eine Tendenz, dass in den letzten Jahren zunehmend jüngere Kinder betroffen sind; möglicherweise sind folgende Ursachen verantwortlich: zum einen Stürze durch den Einsatz von diversen Spielgeräten, z. B. eines Trampolins, aber auch die zunehmende Adipositas bei Kindern. Adipositas führt über das erhöhte Gewicht zu einer Verstärkung des Hypermochlions, das bei dem bekannten Unfallmechanismus des Sturzes auf den Arm mit gleichzeitiger Überstreckung im Ellenbogen zusätzlich zur Wirkung kommt.

\section{Wachstums-Korrekturpotenz}

Der Ellenbogen weist nur ein geringes Wachstumspotenzial auf. Nach dem 6. Lebensjahr kann nur mehr eine sehr geringe Fähigkeit festgestellt werden, Fehlstellungen auszugleichen, sodass sich nicht auf die komplette Korrektur ab dem 6. Lebensjahr verlassen werden kann. Daher sollte eine definitive Stellung ab diesem Alter erzwungen werden. Fehlstellungen in der Frontalebene korrigieren sich nicht, sondern nur Fehlstellungen in der Sagittalebene.

\begin{tabular}{|c|c|c|}
\hline Frakturtyp & Jahre 2009-2019 & Anzahl \\
\hline Supracondylar Fx & $x$ & 94/Jahr \\
\hline Distal radius Fx & $x$ & 64/Jahr \\
\hline Lower leg Fx & $x$ & 60/Jahr \\
\hline \multicolumn{3}{|c|}{$\begin{array}{l}\text { Fx "fracture" } \\
\text { aDie Suche wurde immer mit der zusätzlichen Information kindliche } \\
\text { Fraktur durchgeführt }\end{array}$} \\
\hline
\end{tabular}

\section{Supracondylar humeral fractures in childhood}

The classification of supracondylar humeral fractures in Germanspeaking areas is carried out according to von Laer, which has been appropriated from the $\mathrm{AO}$ system and has the advantage that it can be used to derive the treatment. When indicated immediate surgery is given preference over a delayed treatment. The result is controlled by functional tests directly during the operation. Instability of the fracture and correct placement of the Kirschner $(K)$ wires are challenging. Alternatives are an external fixator and elastically stable intramedullary nailing (ESIN). Concomitant injuries initially affect the median nerve and the brachial artery and secondarily the radial nerve. Lesions of the ulnar nerve are mostly a postoperative complication. The bony consolidation is achieved after 3-4 weeks and afterwards implant removal can be safely carried out. Embedded K-wires and ESIN are removed after 3-6 months, depending on the surgical capacity and complaints of the patient.

\section{Keywords}

Classification · Conservative treatment · Fracture fixation · Bone malposition · Aftercare

Der isolierte Rotationsfehler korrigiert sich, aber weist in der klinischen Praxis immer auf eine Instabilität hin und führt nicht selten über ein ulnares Abkippen zum Cubitus varus. Daher sollte er grundsätzlich vermieden bzw. bei der Fixation sollte kritisch geprüft werden, ob eine Dislokation in einer zweiten Ebene vorliegt. Wachstumsstörungen spielen eine eher untergeordnete Rolle bei der Behandlung der suprakondylären Humerusfraktur im Kindesalter [1, 2, 3].

$\rightarrow$ Cave

Fehlstellungen in der Frontalebene korrigieren sich nicht; Fehlstellungen in der Sagittalebene korrigieren sich bis zum 6/7. Lebensjahr (Abb. 1).

\section{Klassifikationen}

In der Literatur finden sich diverse Einteilungen, wobei in den angloamerikanischen Publikationen die Gartland-Klassifikation [2] am meisten verwendet wird. In den letzten Jahren hat sich in den deutschsprachigen Ländern die Von-Laer-Klassifikation etabliert, die auch von der AO übernommen wurde [1, 5]:

- Typ I: unverschobene Fraktur,

- Typ II: in einer Ebene verschobene Fraktur,

- Typ III: in 2 Ebenen verschobene Fraktur mit Kontakt,

- Typ IV: völlig dislozierte Fraktur ohne Fragmentkontakt.

Diese Klassifikation (Abb. 2) hat den Vorteil, dass sich einheitliche therapeutische Konsequenzen ergeben, die aus den meisten Klassifikation nicht eindeutig abgeleitet werden können. Dies gilt insbesondere, da das Ausmaß der Fehlstellung geschätzt werden muss und damit die eindeutige Zuordnung der Fraktur beurteilerabhängig ist. Ernat et al. (2017) wiesen darauf hin, dass das Behandlungsergebnis v.a. bei der Einteilung nach Gartland nicht vorhergesagt werden kann, obwohl die Autoren in ihrer Studie als einer der wenigen einen prospektiven Ansatz wählten [6]. Die Einteilung nach von Laer/AO ist einfach und nachvollziehbar; ein 

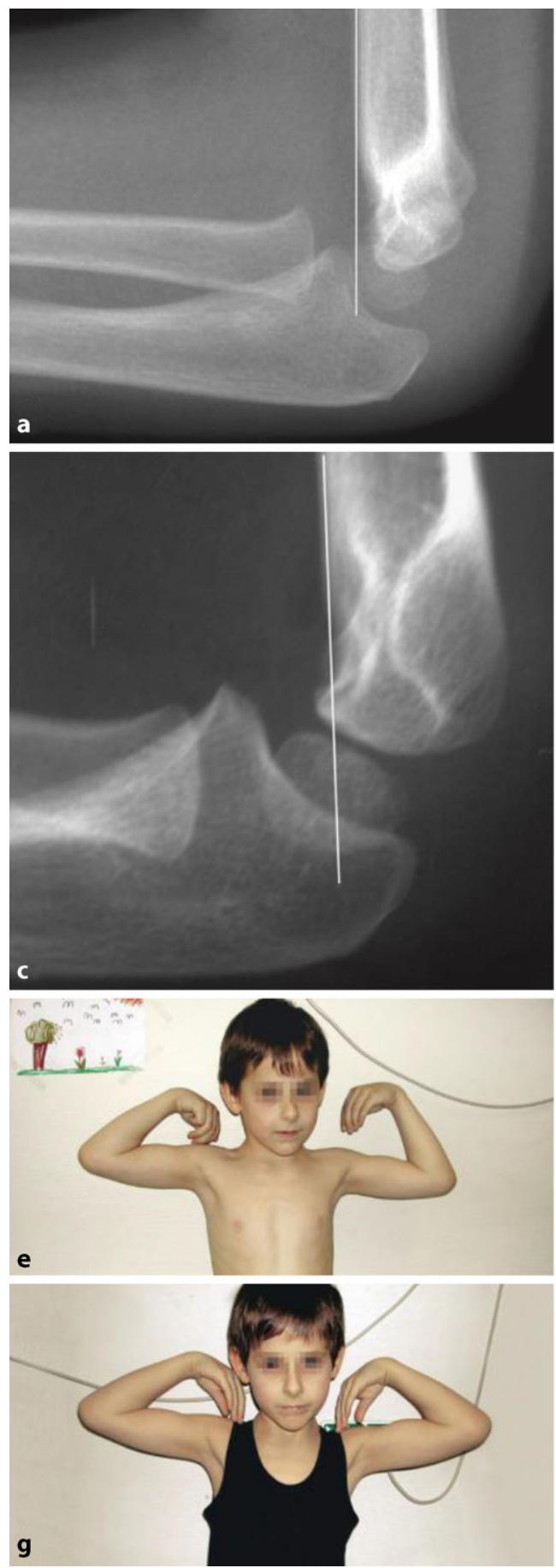
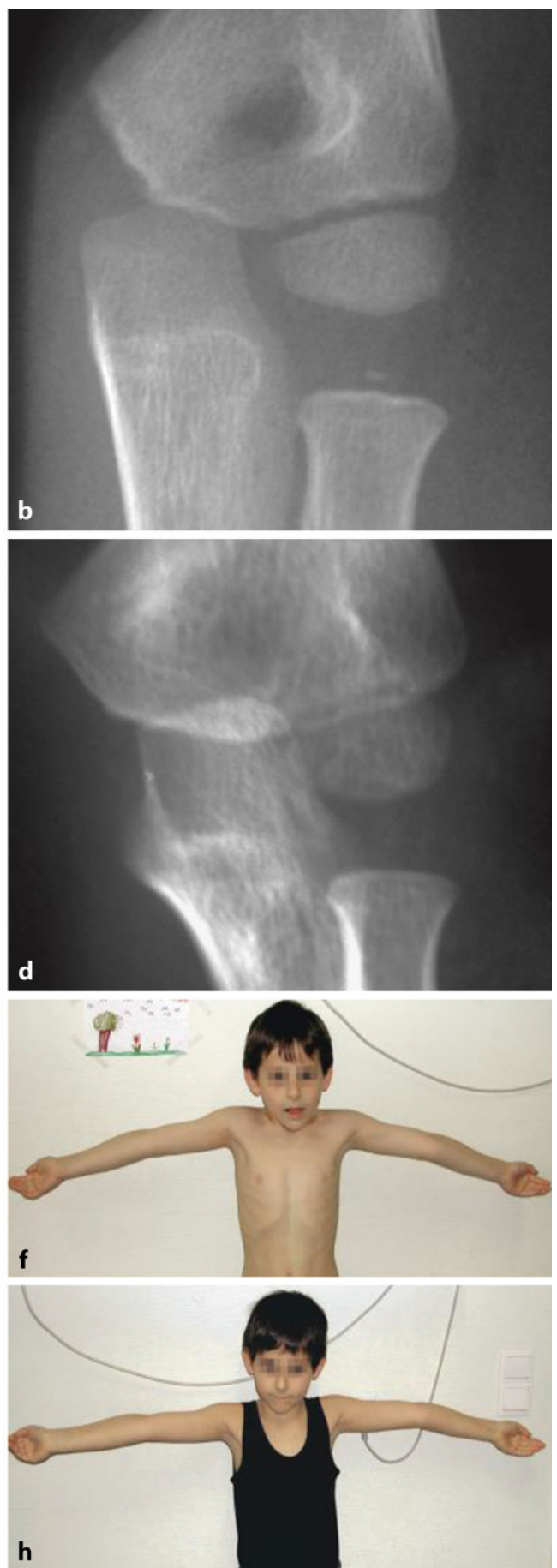

Abb. 1 Beispiel des Remodeling einer Antekurvationsfehlstellung: a, b Ausheilungsbilder mit Antekurvation. c, d Nach erfolgtem Remodeling. Das Capitulum hat sich im Laufe des Wachstums wieder ins Gelenk eingestellt. Die RogersHilfslinie taucht wieder in den mittleren/hinteren Teil des Kerns ein. e, f Klinische Ausheilungsergebnisse: zum Zeitpunkt des Behandlungsabschlusses zeigt der Patient ein Flexionsdefizit (e) und eine leichte Hyperextension (f). $\mathbf{g}, \mathbf{h}$ Bei der Kontrolle nach einem Jahr findet sich eine symmetrische Beweglichkeit. (Aus [4]. Dieser Inhalt ist nicht Teil der Open-Access-Lizenz)
Therapiealgorithmus kann abgeleitet und damit kann auch eine Prognose hinsichtlich des Endergebnisses abgegeben werden. Somit können gleiche Frakturen in verschiedenen Studien miteinander verglichen oder in Reviews zusammengefasst werden, was bisher nicht möglich war.

\section{Radiologie}

Weiterer Vorteil neben der Vergleichbarkeit der Frakturtypen ist die Tatsache, dass der jeweilige Frakturtyp nach Laer/AO immer anhand des Röntgenbilds in 2 Ebenen (a.-p. und seitlich) bestimmt werden kann. Ist die Fraktur initial stark disloziert, kann auf die Aufnahme in der zweiten Ebene primär verzichtet werden, diese sollte dann im OP angefertigt werden.

Einen ersten Hinweis auf das Vorliegen einer Fraktur rund um den Ellenbogen stellt das intrakapsuläre Hämatom dar, das sich radiologisch als "fat pad sign" zeigt. Vor allem das hintere Fat pad sign weist indirekt auf das Vorliegen einer unverschobenen Fraktur hin (Abb. 3). 

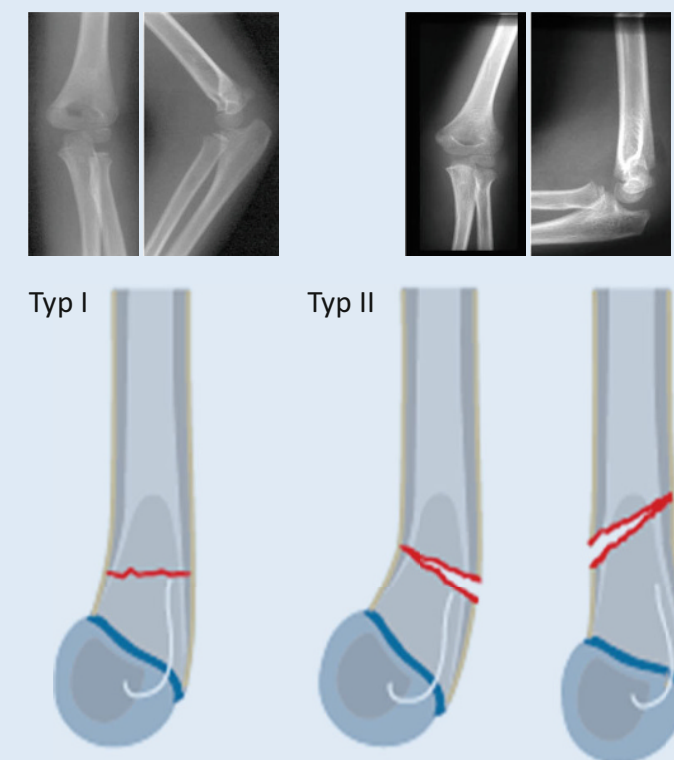

unverschoben
Typ II

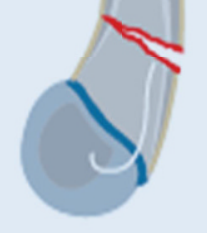

Flexionsverletzung Rekurvation

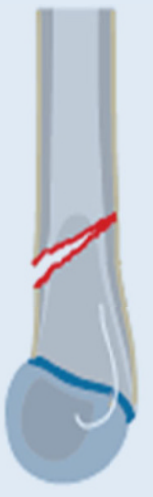

Extensionsverletzung Antekurvation
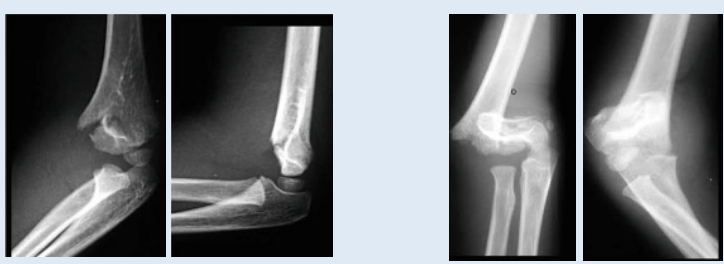

Typ III

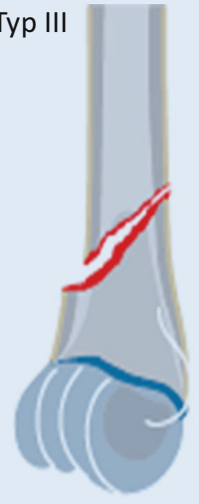

Verschiebung in mindestens 2 Ebenen
Typ IV

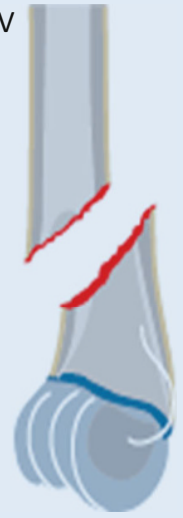

Völlig disloziert

Abb. 2 ム Frakturklassifikation. (Aus [4]. Dieser Inhalt ist nicht Teil der Open-Access-Lizenz)

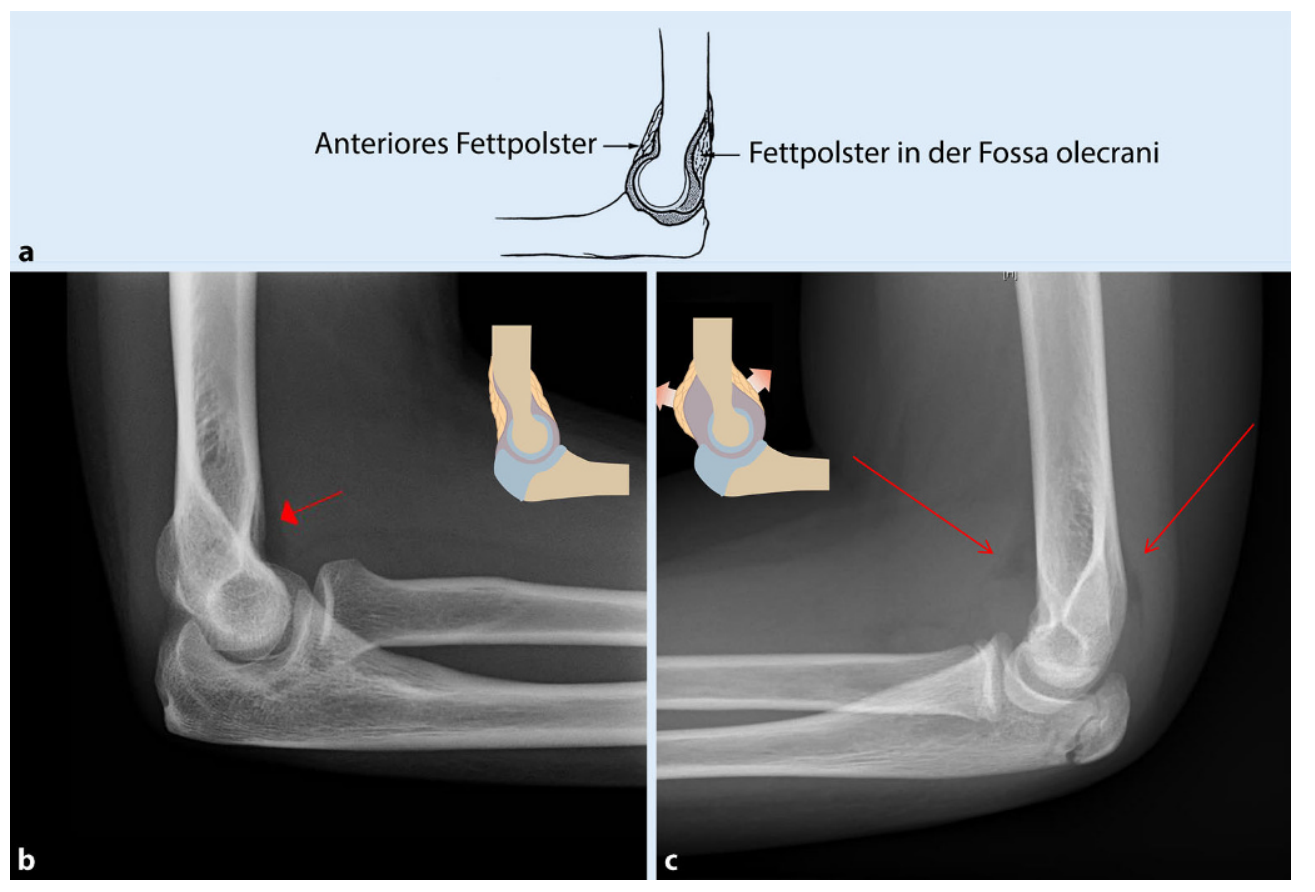

Abb. $3<$ Vorderes und hinteres "fat pad sign". a Schematische Darstellung, b Röntgenaufnahme mit Darstellung des vorderen Pad sign (Pfeil), c Röntgenaufnahme mit Darstellung des vorderen und hinteren Pad sign (Pfeile). (Aus [4]. Dieser Inhalt ist nicht Teil der Open-Access-Lizenz)
Um Fehlstellungen zu analysieren, werden das a.-p.- und das seitliche Röntgenbild herangezogen. Die folgenden radiologischen Hilfslinien können verwendet werden (Abb.4; [1]):

- Diaphysen-Epiphysen-Winkel: Der Winkel zwischen Humeruslänge und Epiphysenfugenachse soll im seitlichen Strahlengang zwischen 30 und $40^{\circ}$ betragen. Winkel $<30^{\circ}$ und $>40^{\circ}$ sprechen für Rekurvations- bzw. Antekurvationsfehlstellungen (Abb. 4a).

- Baumann-Winkel: Der Baumann-Winkel wird zur Abschätzung der Achse des Ellbogengelenks genutzt, insbesondere wenn der Arm nicht gestreckt und supiniert werden kann. Er ist definiert als der Winkel zwischen der Epiphysenachse der lateralen Kon- 


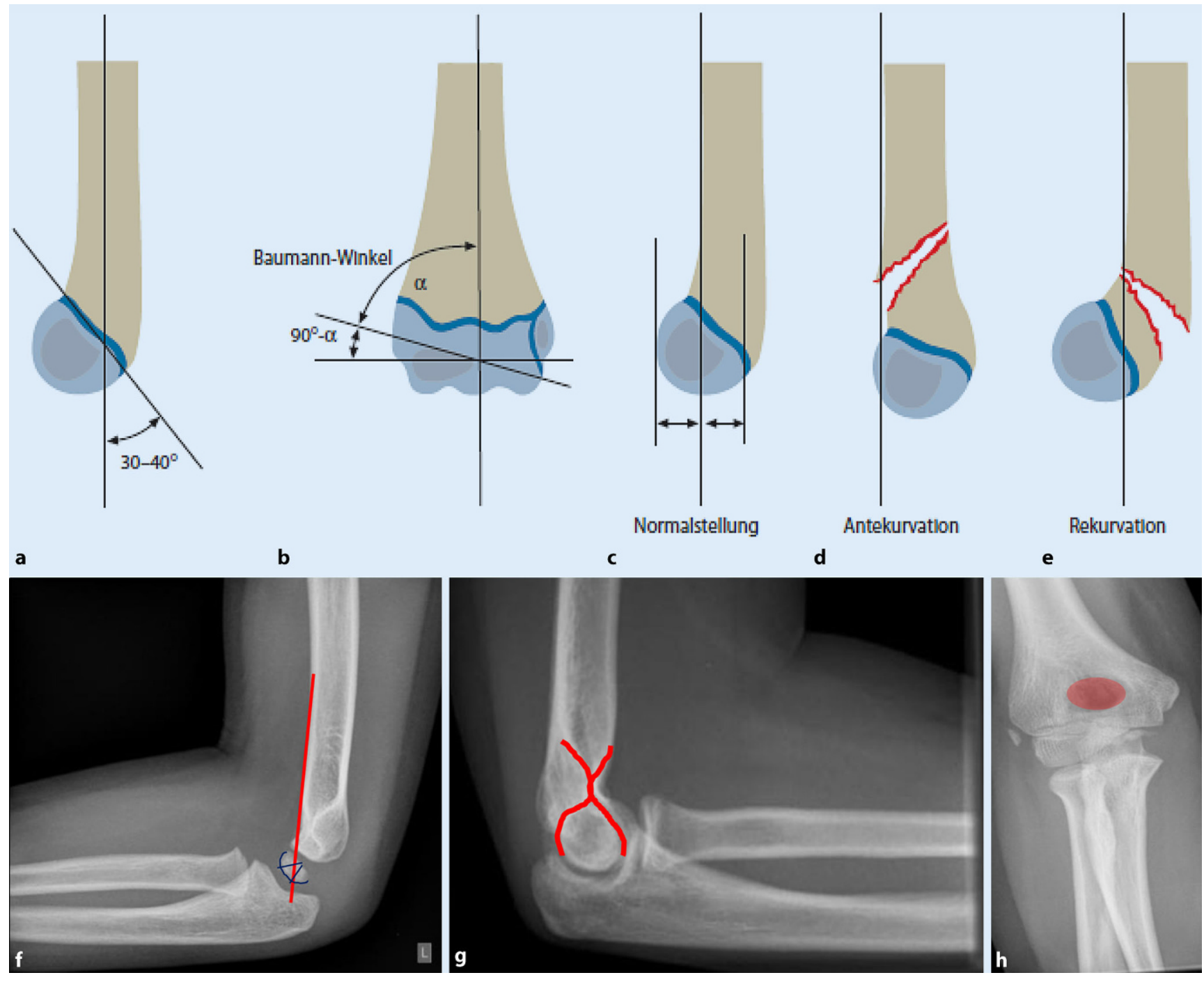

Abb. $4 \Delta$ a-e Schematische Darstellung der radiologischen Hilfslinien. Erklärungen s. Text.f Korrekte Rogers-Hilfslinie bei nichtgebrochenem Ellenbogen im Nativröntgenbild. g, $\mathbf{h}$ Sanduhrzeichen als Hinweis auf einen intakten Ellenbogen. (Aus [4]. Dieser Inhalt ist nicht Teil der Open-Access-Lizenz)

dylen und einer Senkrechten zur Humeruslängsachse. Zumeist wird der Baumann-Winkel jedoch als Winkel zwischen Humeruslängsachse und Epiphysenachse des lateralen Condylus beschrieben. Wird dieser Winkel von $90^{\circ}$ subtrahiert, ergibt sich die Achse des Ellbogengelenks (Abb. 4b).

- Hilfslinie nach Rogers: Diese Hilfslinie wird im Alltag am meisten genutzt, da sie einfach zu handhaben und klinisch gut verwertbar ist. Diese entspricht im seitlichen Strahlengang einer Tangentiale entlang der vorderen Humeruskortikalis, deren Verlängerung im Normalfall (Abb. 4c) das Capitulum humeri am Übergang vom mittleren zum hinteren Drittel schneidet. Bei der Antekurvationsfehlstellung schneidet die Linie im vorderen Drittel oder außerhalb (Abb. 4d). Im Fall einer Rekurvationsfehlstellung schneidet sie im hinteren Drittel oder außerhalb (Abb. 4e).

\section{Therapie}

\section{Besondere Herausforderungen}

Die besonderen Herausforderungen der Behandlung lassen sich auf folgende Faktoren zurückführen:

- Dynamik der Fraktur,

- Schwierigkeit der Beurteilung des Röntgenbilds,

- dass die Retinierbarkeit der Fraktur ebenfalls nicht einfach ist, insbesondere die Stabilität und die radiologische Beurteilung der intraoperativen Fixation der Fraktur.

Die frakturbezogene Problematik ergibt sich daraus, dass es sich seltener Quer-, sondern meist um Schrägfrakturen handelt. Diese bereiten oftmals mehr Probleme, sie in der Reposition zu halten zumal das distale Fragment sehr klein sein kann. Die vorhandene Muskulatur trägt dazu bei, dass die Fragmente unter Zug kommen 


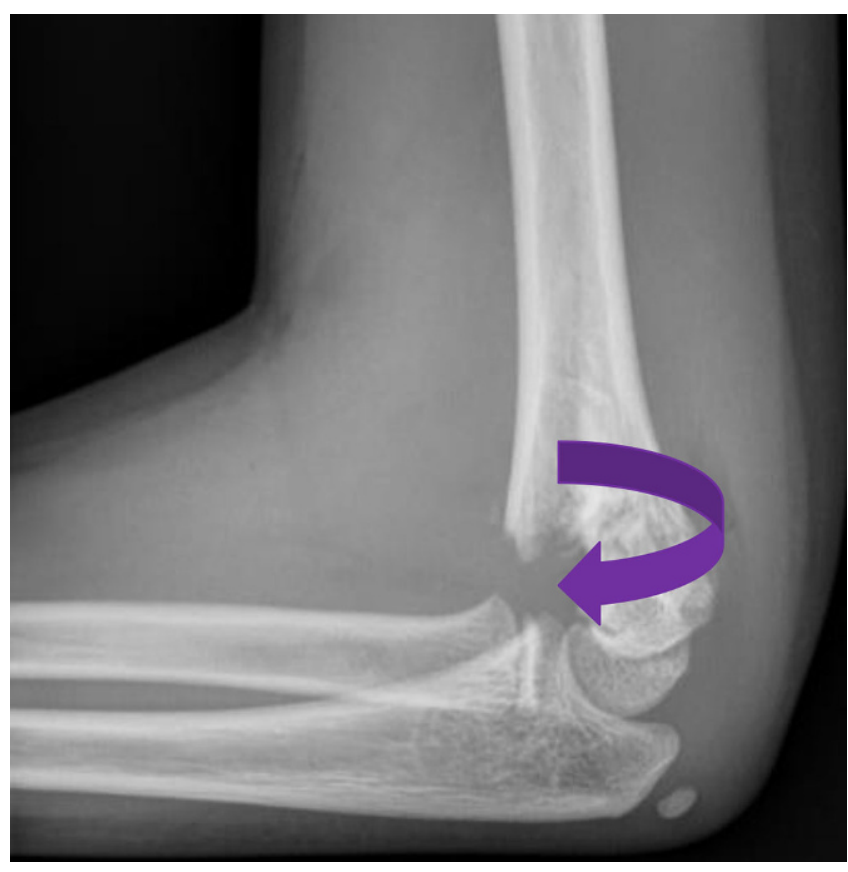

Abb. 5 A Rotationssporn im radiologischen seitlichen Bild

und sich während der Behandlung bzw. unter der Reposition wieder verschieben $[2,3]$.

\section{Merke}

Proximal weist die Fraktur eine Außenrotationstendenz auf, und distal wird sie durch den Muskelzug nach innen rotiert.

Der schon oben erwähnte Rotationsfehler ist abhängig von Drehpunkt und Ausmaß der Fehlstellung, wobei der Rotationsfehler an sich nicht das Problem darstellt, sondern das Verkippen der Fragmente in einen Cubitus varus und nur äußerst selten in einen Cubitus valgus. Cubitus varus und Cubitus valgus korrigieren sich nicht. Andere Dislokationsformen beinhalten eine Verschiebung in der Sagittalebene. Diese kann altersbedingt korrigiert werden (s. Abschn. „Wachstumskorrekturpotenzial").

\section{- Cave}

Cubitus varus und Cubitus valgus können sich im Laufe des Wachstums nicht korrigieren.

Die Abhängigkeit des Drehpunkts und des Verlusts der Kontaktfläche sind maßgebliche Ursachen für die in der Folge entstehenden sekundäre Dislokation und anschließenden klinischen Cubitus varus, selten Cubitus valgus. Die Schwierigkeit der Fixation der kindlichen Knochen nach suprakondylärer Fraktur besteht darin, dass bei einem Drehfehler ab $20 \%$ bereits die Fragmentkontaktfläche um $50 \%$ verringert ist (Abb. 5, 6) und damit die Fixation durch Kirschner(K)-Drähte und das korrekte Einbringen derselben schwieriger ist als bei korrekter Reposition und vollständiger Kontaktfläche $[3,7]$. Diese Verdrehung kann aber nur schlecht im Röntgenbild erkannt werden, wobei dies vom Strahlengang abhängig ist. $10^{\circ}$

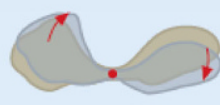

$20^{\circ}$

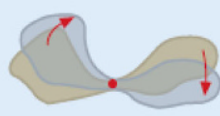

$30^{\circ}$

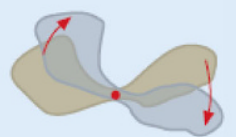

$40^{\circ}$

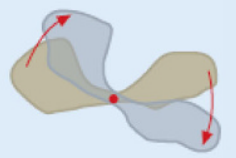

$50^{\circ}$

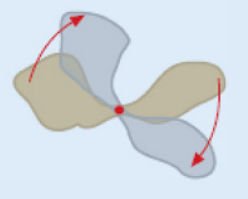

Abb. $6<$ Die Kontaktfläche der Fraktur zum Einbringen der Kirschner-Drähte ist vom Ausmaß des Drehfehlers abhängig, der radiologisch erst $\mathrm{ab} 20^{\circ}$ sicher erkannt werden kann (ulnoradialer Strahlengang). Ein Drehfehler von $20^{\circ}$ bedeutet bereits einen erheblichen Kontaktflächenverlust. (Aus [4]. Dieser Inhalt ist nicht Teil der Open-Access-Lizenz)

\section{$>$ Cave}

Im ulnoradialen Strahlengang ist der Rotationsfehler bereits $a b 20^{\circ} \mathrm{zu}$ erkennen. Im radioulnaren Strahlengang, d.h., im klassischen a.-p.-Bild ist ein Rotationsfehler von $20^{\circ}$ nicht zu erkennen.

Klinisch ist der isolierte Rotationsfehler nicht messbar. Im radiologischen Bild ist der Rotationsfehler nur am Kalibersprung, damit ist der Durchmesser beider Frakturenden gemeint, oder am Rotationssporn zu erkennen. Daraus kann abgeleitet werden, dass das Endergebnis - insbesondere intraoperativ - klinisch und nicht nur anhand des Röntgenbilds geprüft werden sollte. Die Beurteilung des Repositions- und Retentionsergebnisses ist am Ende der Operation zu dokumentieren.

\section{Dichtig}

Intraoperative klinische Prüfung: Eine symmetrische Ellbogenachse schließt ein seitliches Abkippen aus. Eine Flexion bis $120^{\circ}$ eine AK/ Antekurvation) und einen RF (Rotationsfehler), sowie eine Extension in Nullstellung schließt eine RK-Fehlstellung (Rekurvation) aus.

Die Frakturen sind vermehrt instabil, wenn Sie eine Trümmerzone aufweisen, die aber auch durch fehlerhafte, vermehrte Bohrversuche entstehen kann. Hier kann der Fixateur externe eine hilfreiche Option darstellen. Nach Meinung der Autoren stellt dieser initial bei älteren und hohen (proximalen) suprakondylären Oberarmfrakturen eine gute Option dar. Der Fixteur externe ist bei kleinen Kindern und distalen Frakturen mit Repositionsschwierigkeiten bzw. im Rahmen von Revisionen Mittel der Wahl.

Da Frakturen nicht gerade Bruchlinien aufweisen, können kleine Fragmente oder Bruchkanten Hindernisse bei der Reposition darstellen. Diese erschweren die Reposition in korrekter Stellung. Hier kommt die „Joy-stick“-Technik zur Anwendung. Zusätzlich bietet 

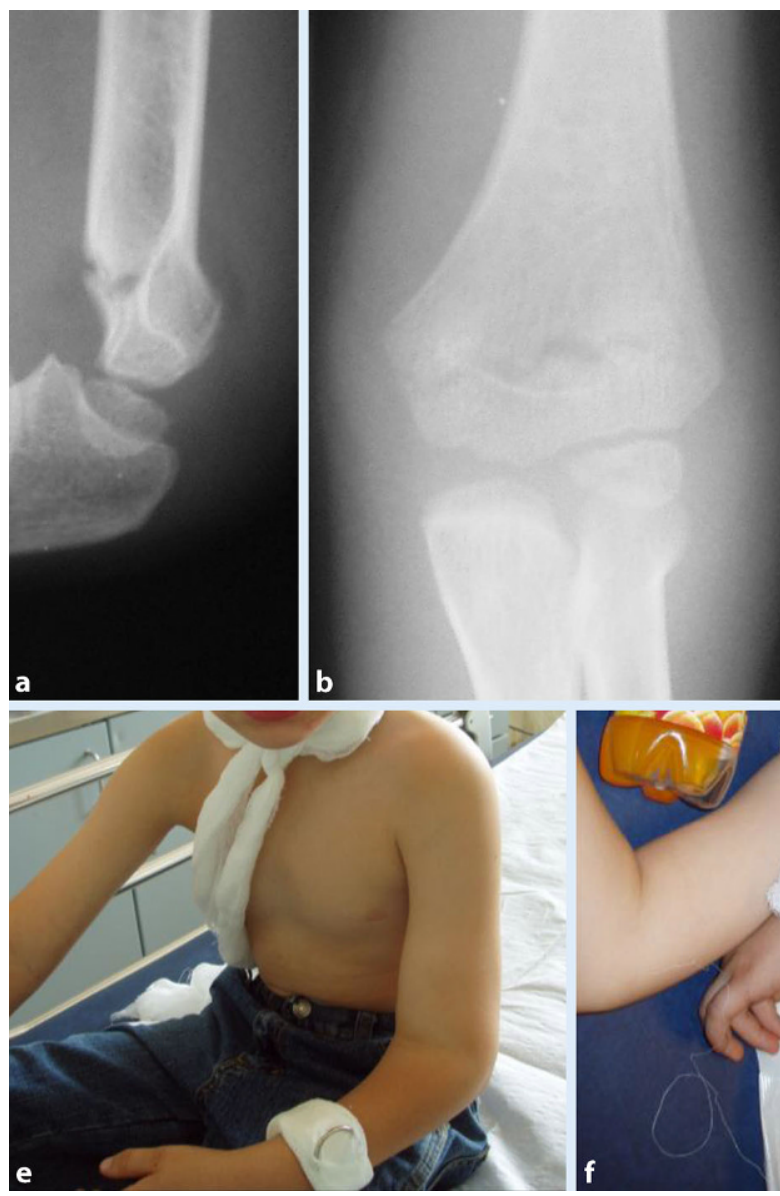
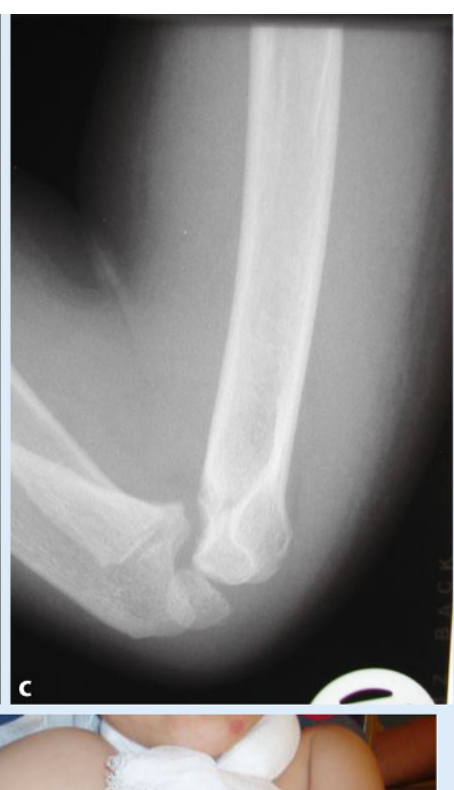

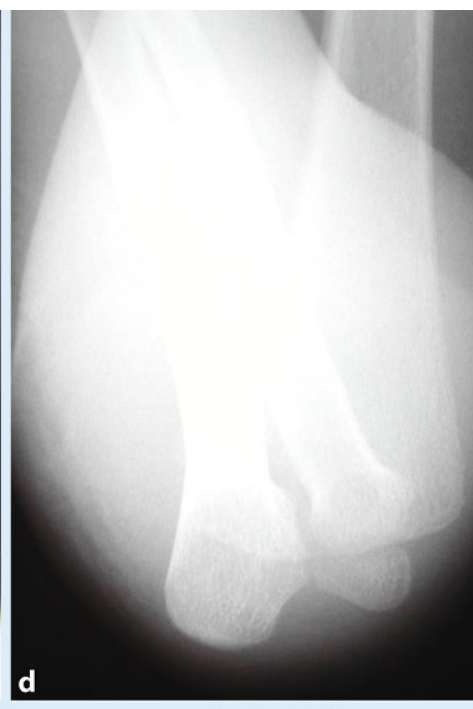

Abb. 7 A Typische Antekurvationsfehlstellung mit Anlage eines Blount-Verbands. a, b Unfallbild einer Typ Il nach VON Laer/Ao (Extensionsfehlstellung, c, d Gelungene Redression nach Blount-Schlinge, e, f klinische Anlage, $\mathbf{g}$ schematische Darstellung der Anlage einer Blount-Schlinge

der Fixateur externe den Vorteil, einen stabilen definitiven Abstand bei Trümmerzonen zur Verhinderung einer Achsenabweichung zu gewährleisten.

Die Problematik der suprakondylären Oberarmfraktur kann wie folgt zusammengefasst werden: Grundproblem ist die Frakturinstabilität, die durch den Muskelzug verursacht wird. An sich ist die Fraktur gut zu reponieren (>70-95\% geschlossene Reposition, je nach Publikation), aber das Repositionsergebnis ist radiologisch nicht immer sicher beurteilbar, sodass Fehlstellungen nicht erkannt werden. Die Fraktur zeigt eine schlechte Retinierbarkeit (offene Fugen). Trümmerzonen oder ausgebrochene Fragmente - auch unter der Operation durch vermehrte Repositionsversuche oder frustrane Spickdrahteinbringungen - können die Retention in ihrer Stabilität erheblich gefährden.

\section{Therapieziel}

Das Therapieziel der suprakondyläre Humerusfrakturen kann wie folgt definiert werden:

- keine Funktionseinschränkungen und

- kein kosmetisches Defizit.
Ersteres bedeutet keine bleibenden Nervenläsionen und keine Funktionsdefizite durch bleibende Achsenfehlstellungen. Die Kosmetik beinhaltet seitengleiche Armachsen.

Weiteres Therapieziel ist es, dem Kind eine möglichst rasche Mobilität bei geringem Aufwand zu gewähren.

$>$ Cave

Die Beurteilung der Achse zur Dokumentation des Endergebnisses kann nur bei voller Extension geprüft werden. Weist das Kind ein Extensionsdefizit auf, wird eine valgische Armachse vorgetäuscht.

Um die Umsetzung der Therapieziele zu erreichen, sind folgende Aspekte essenziell:

- primär korrekte Beurteilung des Röntgenbildes und in der Folge die daraus abzuleitende Behandlung,

- korrekte Festlegung der Therapie (nur Ruhigstellung, Redression oder Reposition und Fixation),

- möglichst keine Verfahrenswechsel,

- primär eine definitive Behandlung anstreben,

- klinische intraoperative Beurteilung des Repositionsergebnisses,

- Retention, die bis zur Konsolidation stabil ist. 


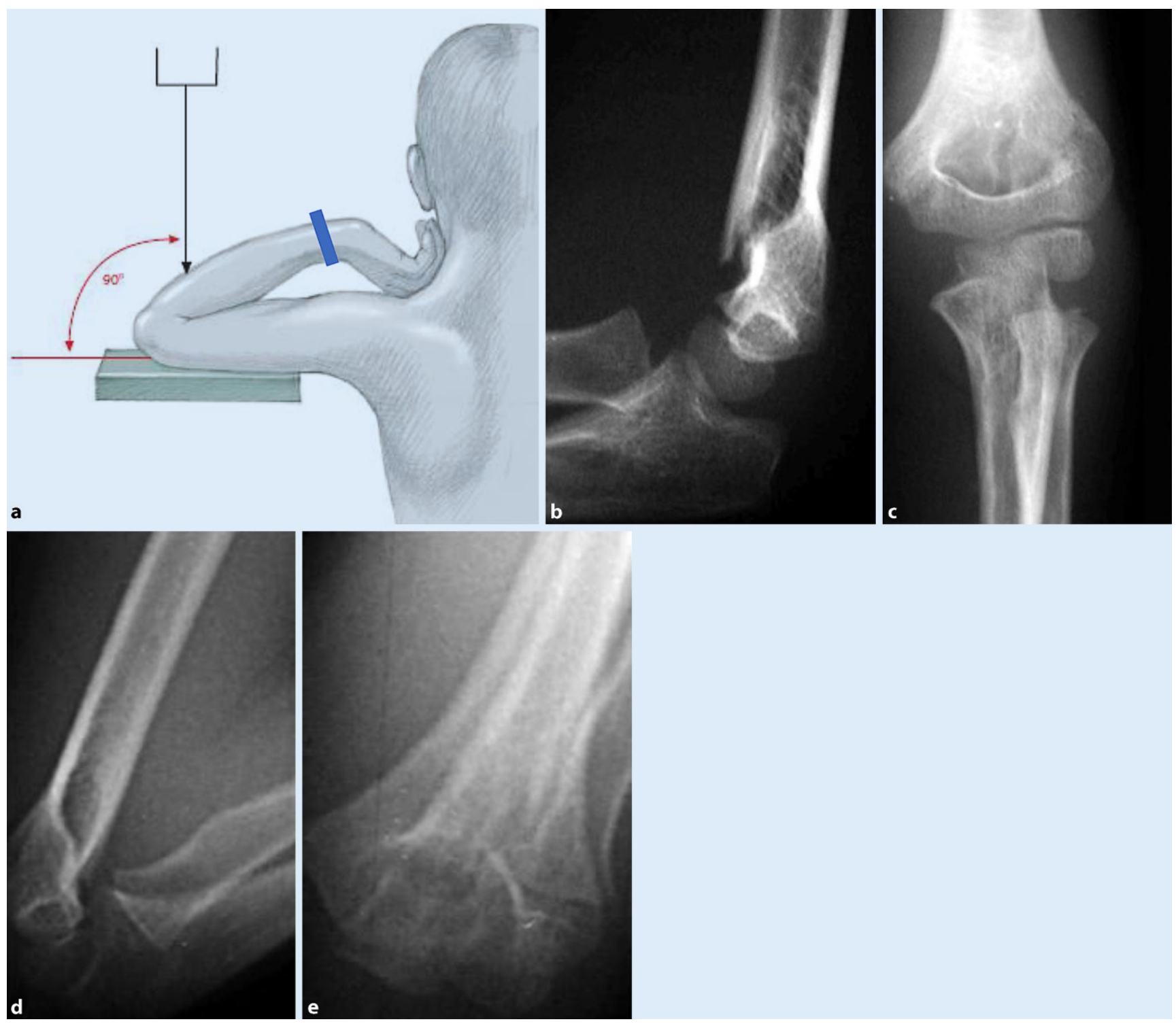

Abb. 8 A Radiologische Technik bei angelegter Blount-Schlinge a.-p. und seitlich. Wurde dem Patient eine Blount-Schlinge angelegt, kann keine a.-p.-Aufnahme angefertigt werden, da sich sonst die Redression aufhebt. In diesen Fällen ist eine axiale Darstellung (a) notwendig, um sekundäre Dislokationen auszuschließen. b, c Unfallbild einer Antekurvationsfehlstellung, $\mathbf{d}$, e radiologische Kontrolle einer erfolgreichen Redression durch eine Blount-Schlinge. (Aus [4]. Dieser Inhalt ist nicht Teil der Open-Access-Lizenz)

\section{Konservative Therapie}

\section{Indikationen}

Die konservative Therapie ist bei Typ-I-Frakturen indiziert. Typ-IIFrakturen weisen eine Re- bzw. Antekurvationsfehlsstellung auf. Antekurvationsfehlstellungen werden insbesondere in Österreich und der Schweiz durch Ruhigstellen in der Blount-Schlinge ("Cuff-and-collar"-Verband) behandelt, die eine Redressionsmethode darstellt. Die Rekurvationsfehlstellung, die weitaus seltener vorkommt, kann mithilfe dieser Methode nicht behandelt werden (Abb. 7).

Allerdings sollte beachtet werden, dass Kinder, die keine Spontankorrektur mehr aufweisen (nach dem 6. Lebensjahr) definitiv zu reponieren sind und dies nicht mithilfe des Redressionsverfahrens erfolgen sollte. Die Reposition sollte aktiv unter Narkose vorge- nommen werden. Dies zieht meist eine definitive Versorgung nach sich (Osteosynthese; $[1,2,3,7]$ ).

\section{Anlage einer Blount-Schlinge (Cuff-and-collar-Verband)} Zunächst sind die geforderten $110^{\circ}$-Flexion in der Blount-Schlinge nicht zu erzwingen, sondern der Arm wird in $90^{\circ}$-Flexion ruhiggestellt. Den Eltern des Patienten wird gezeigt, wie sie den Verband innerhalb des schmerzfreien Bereichs nachziehen können, sodass letztlich die Spitzwinkelstellung von $110^{\circ}$ erreicht wird. Wichtig ist die Wiedereinbestellung des Patienten nach 3 bis 5 Tagen, um die Anlage und auch die Frakturstellung zu kontrollieren (Abb. 7; $[2,3])$. 


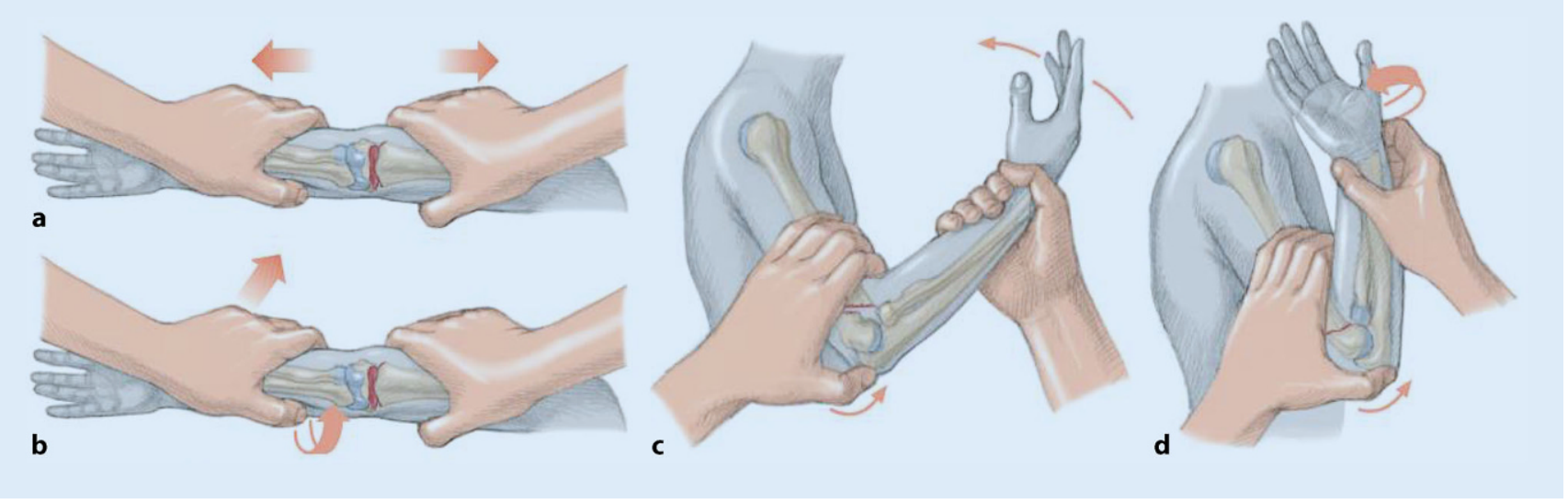

Abb. 9 ム Repositionstechnik der suprakondylären Oberarmfraktur. a Zunächst wird die Fraktur unter Längszug gebracht; b anschließend Korrektur einer Achsfehlstellung in der Frontalebene und ggf. eines Rotationsfehlers; c Beugung meist in Pronation unter Druck des Daumens auf das Olecranon, um das distale Fragment zu reponieren; $\mathbf{d}$ korrekte Reposition des distalen Fragments unter Daumendruck. (Aus [4]. Dieser Inhalt ist nicht Teil der Open-Access-Lizenz)

\section{Merke}

Die Blount-Schlinge (Cuff-and-collar-Verband) stellt ein Verfahren dar, das die Stellung indirekt korrigiert. Ein sofort angefertigtes Röntgenbild zeigt daher oftmals nicht die Redression, die sich erst im Laufe der Zeit einstellt. Eine Kontrolle der Stellung sollte nach 3 bis 5 Tagen angestrebt werden.

Mit diesem Vorgehen kann eine Volkmann-Kontraktur, die in der Literatur als Sekundärschaden einer supracondylären Oberarmfrakturversorgung in Einzelfällen beschrieben wurden, sicher vermieden werden, da Eltern die gewünschte Stellung meist durch sachtes Vorgehen erreichen und diese nicht im geschwollenen Zustand des Arms und unter Schmerzen in der Ambulanz provoziert wird. Ein Problem bei der Anlage von Blount-Schlingen stellt die radiologische Diagnostik dar. Oftmals wird die Schlinge zum Röntgen irrtümlich abgenommen. Dies ist nicht nötig (Abb. 8). Grundsätzlich reicht für die Stellungskontrolle der TypII-Frakturen ein seitliches Röntgen in angelegter Redression, für den Ausschluss von sekundären Dislokationen ist die Anfertigung einer a.-p.-Aufnahme notwendig.

Sollte die indirekte Redression nicht ausreichen, können nach 3 bis 5 Tagen immer noch eine Reposition und definitive Versorgung in Narkose durchgeführt werden. Muccioli et al. beschrieben in einer Arbeit aus dem Jahr 2017, dass dieses Vorgehen kostengünstig ist. Es setzt aber eine gute Kooperation mit dem Nachbehandler voraus, wenn dies das Krankenhaus nicht durchführen darf. In Österreich und der Schweiz ist diese Methode weit mehr verbreitet [8].

Wie im Abschn. „Epidemiologie“ bereits angesprochen, stellt die zunehmende Adipositas im Kindesalter ein Problem dar, das den Chirurg zusätzlich herausfordert. Li et al. berichteten, dass übergewichtige Kindern 4-mal so häufig operativ versorgt werden müssen wie normalgewichtige Kinder [9]. Der scheinbar nach unten rückende Altersgipfel [9] stellt eine zusätzliche Herausforderung dar, da diese Altersgruppe eine erhöhte Komplikationsrate aufweist und die Retention bzw. Fixation zusätzlich durch das geringere Knochenvolumen und den großen Weichteilmantel erschwert wird.

\section{Operative Therapie}

An osteosynthetischen Optionen stehen K-Draht, Fixateur externe und elastisch stabile intramedulläre Nagelung (ESIN) zur Verfügung. In deutschsprachigen Ländern wird die K-Draht-Osteosynthese am meisten angewendet. Grundsätzlich sollte jeder Operateur alle genannten Varianten der Frakturfixation beherrschen.

\section{Reposition}

Bevor das operative Prozedere beginnt, muss die Fraktur reponiert werden. Hier ist es essenziell, zunächst einen Längszug auszuüben, der einige Zeit gehalten werden sollte, bevor mit dem Repositionsmanöver begonnen wird. Hier können verschiedene Techniken zum Einsatz kommen. Da aber das distale Fragment meist dorsal liegt, sollten die Weichteile durch den Längszug ausreichend gedehnt worden sein, und es sollte eine Diastase zwischen den Fragmenten bestehen. Deshalb kann das Fragment durch Druck mit dem Daumen auf das Olecranon auf den proximalen Schaft reponiert werden (Abb. 9). Diese Maßnahme ist nur erfolgversprechend, wenn das Fragment distal nicht hinter dem proximalen Anteil liegt. Bei distal posteriorer Fehlstellung muss eher der proximale Anteil durch Druck von ventral in Höhe des distal dorsal gelegenen Fragments gebracht werden. Voraussetzung ist die durch den Längszug erreichte Diastase zwischen den Fragmenten. Die geschlossene Reposition geling in $>70-95 \%$ der Fälle.

In manchen Kliniken werden die Patienten zur Oberarmfrakturversorgung in Bauchlage operiert. Vorteil dieser Lagerung ist, dass die Reposition leichter gelingt, da der proximale Anteil des Arms durch die Armlehne fixiert wird, der distale Anteil durch den Zug des Eigengewichts des Armes eine Diastase in der Frakturzone bewirkt und die Reposition sich beim Beugen des Arms meist von allein einstellt. Nachteil dieser Lagerung ist, dass bei Schwierigkeiten in der Reposition die mit der Rückenlage verbundene Flexibilität aufgegeben wurde. Die Rückenlage bietet dem Operateur gleichzeitig eine dorsale Zugangsmöglichkeit zum Ellenbogen, falls offen reponiert werden muss, sodass die Notwendigkeit zweiseitigen Zugangs entfällt. Die Bauchlage ist aber aus anästhesiologischer Sicht mit höheren Komplikationen ver- 

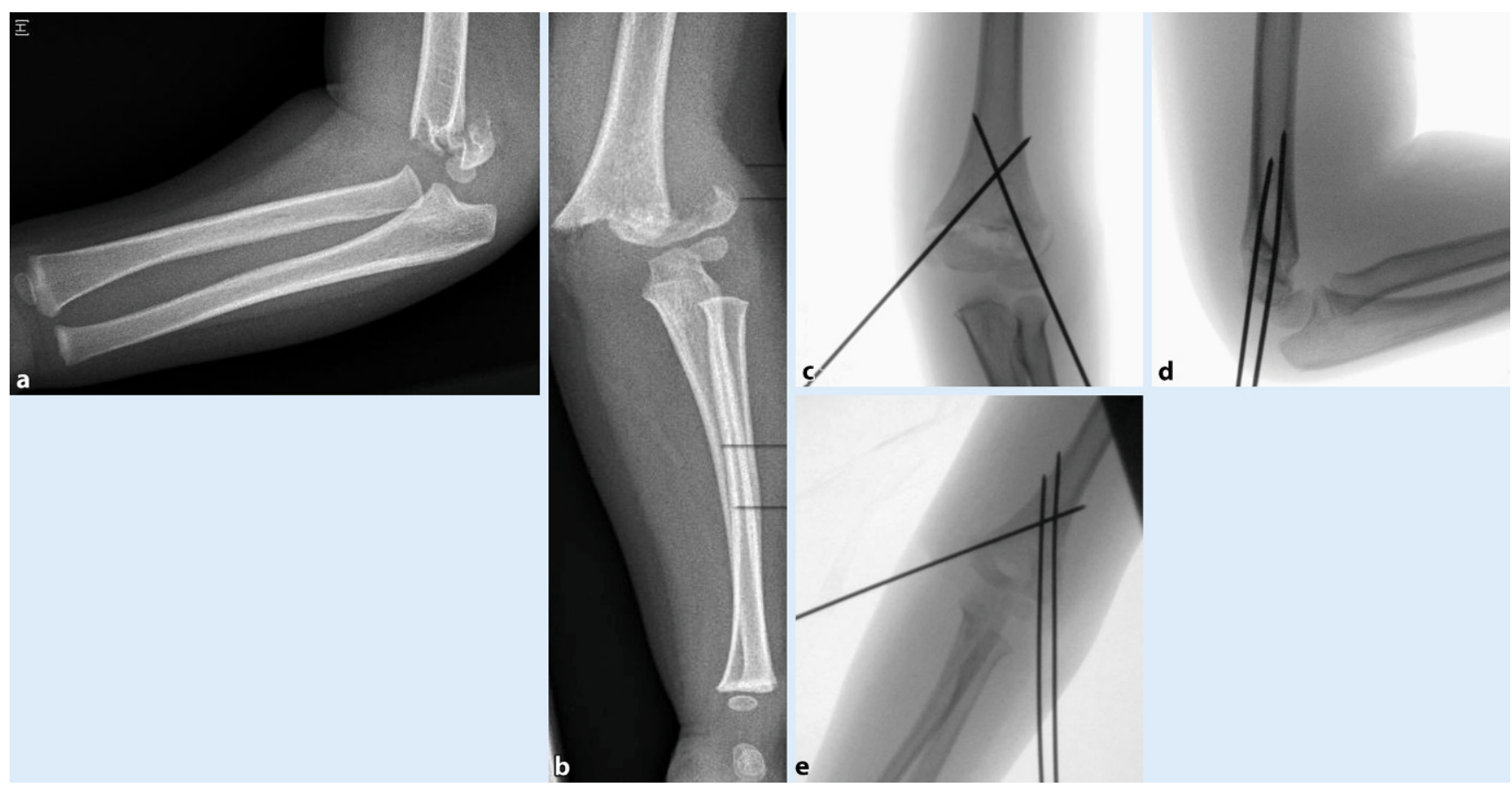

Abb. 10 A Klinisches Beispiel Osteosynthese mithilfe von 3 K-Drähten. a, b Unfallbilder, c-e intraoperativer Situs

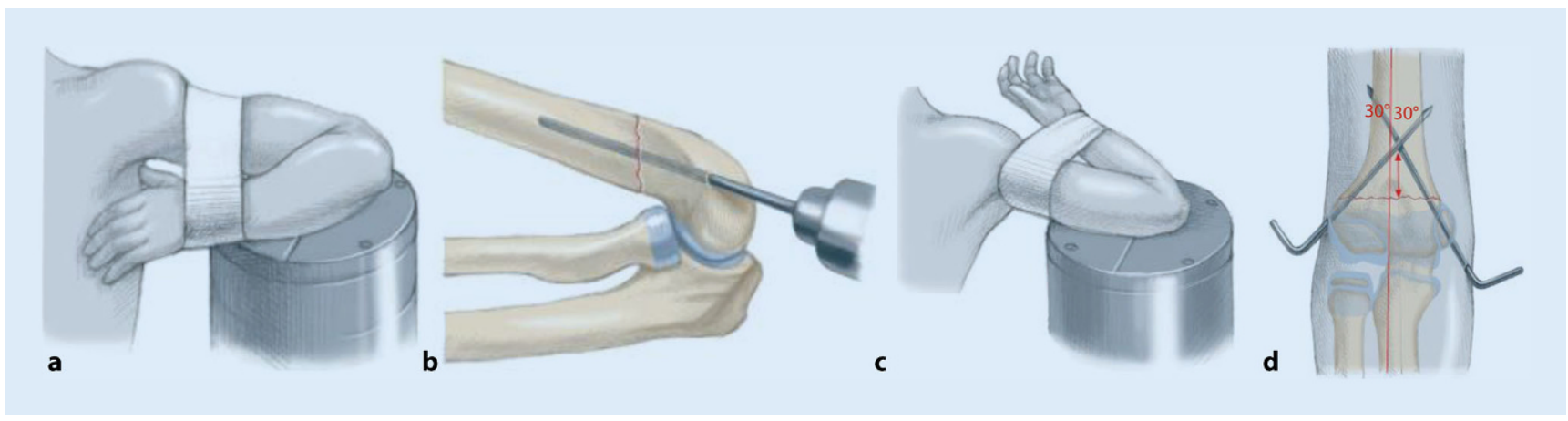

Abb. 11 ॥ Kirschner-Draht-Osteosynthese. Im Rahmen der gekreuzten Variante muss beim Einbringen des medialen Drahts darauf geachtet werden, dass der N. ulnaris geschont wird. Es gilt: Ulnaren Draht von ventral vor der Epikondyle nach dorsal ziehend einbringen und den radialen K-Draht vice versa. Kreuzungspunkt ist proximal der Frakturlinie. Drahtdurchmesser: 1,6-2,0 mm (altersabhängig). a Radioulnarer Strahlengang, b Drahteinbringung in der seitlichen Ebene mittig, culnoradialer Strahlengang, d korrektes Einbringen im ap Strahlengang der Drähte. Kreuzungspunkt oberhalb derFraktur. (Aus [4]. Dieser Inhalt ist nicht Teil der Open-Access-Lizenz)

bunden oder wird sogar von manchen Kliniken, da das Kind als per definitionem nach einem stattgehabten Trauma als nichtnüchtern einzustufen ist, abgelehnt. Meist wird also die Rückenlage bevorzugt [3].

\section{Osteosynthese}

Kamara et al. stellten Ergebnisse einer Studie aus dem Jahr 2018 vor und folgerten [10]:

- Bei Frakturen, die im proximalen Teil der Metaphyse lokalisiert sind, erweist sich die ESIN-Technik als die beste Versorgungsvariante.

- Für mittig in der Metaphyse gelegene Frakturen erzielt die gekreuzte K-Draht-Osteosynthese (2 Pins von lateral und 1 Pin von medial) bessere Resultate als die gekreuzte Spickung mithilfe von 2 Drähten.

- Dasselbe gilt für ganz distal gelegene Frakturen.

Dieser Algorithmus wird im deutschsprachigen Raum bereits seit mehreren Jahren angewandt.

Kirschner-Draht-Osteosynthese. Die korrekte Fixation der Fraktur mithilfe von K-Drähten ist wichtiger als die Zahl der eingebrachten Drähte. Eine absolute Stabilität wäre nur wünschenswert, wenn gipsfrei nachbehandelt würde. Drei Drähte gewährleisten eine biomechanisch höhere Stabilität als 2, und auch 4 Drähte sind besser als 2 bis 3 . Aber bei korrektem Einbringen sind 2 Drähte völlig ausreichend - abgesehen davon, dass diese hohe Zahl der Drähte altersabhängig schwierig zu platzieren ist und insbeson- 

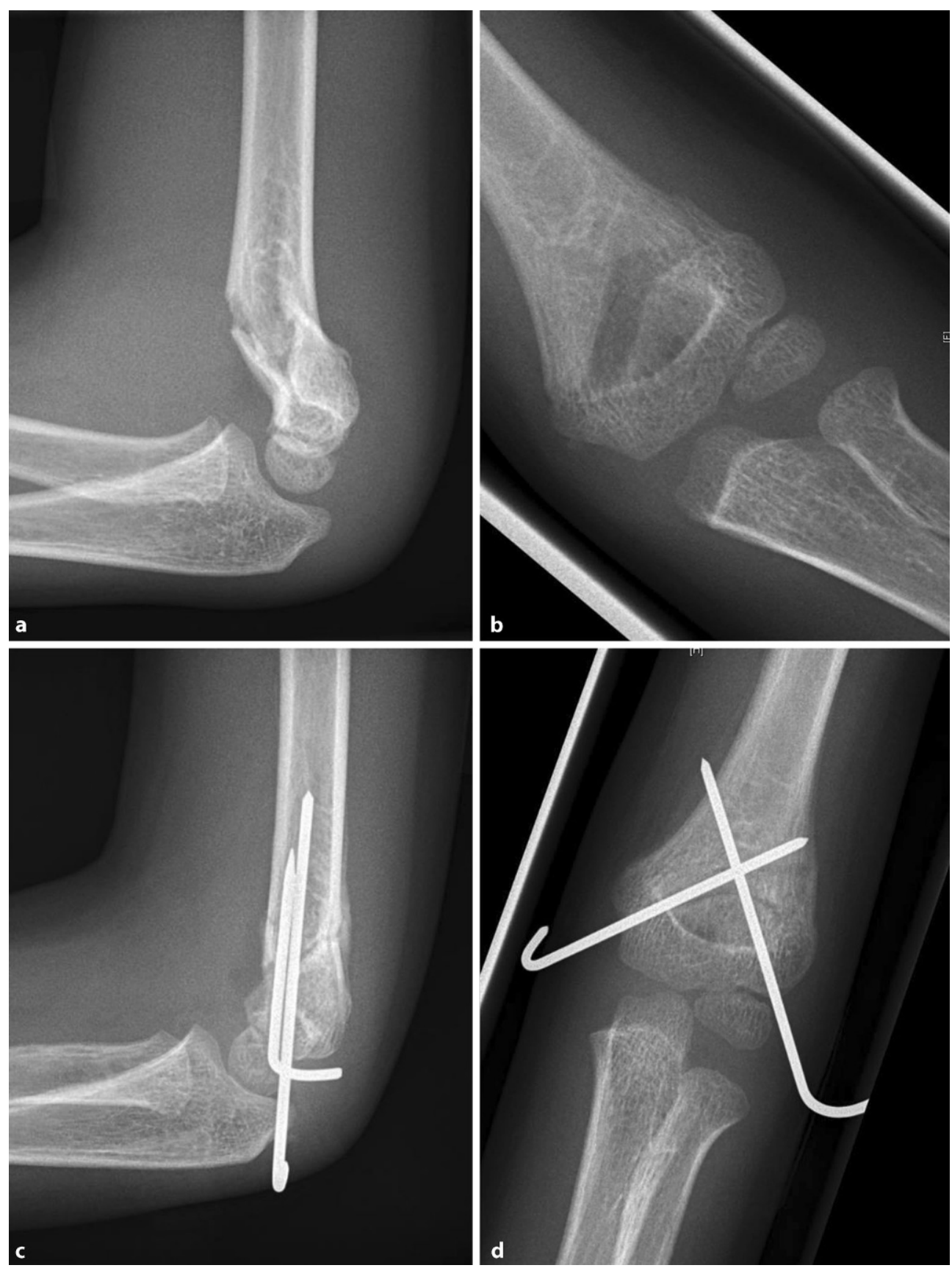

Abb. $12 \triangleleft$ a, b Unfallbild einer typischen Extensionsfraktur bei einem 9-jährigen Jungen, Typ-II-Fraktur nach von Laer/AO, wobei altersentsprechend die Fehlstellung nicht belassen werden kann. c, d Reposition mithilfe von 2 gekreuzten Kirschner-Drähten und Belassen der Drähte außerhalb des Hautniveaus

dere beim kleinen Kind mit viel knorpeligen Anteil in Augen der Autoren vermieden werden sollte (Abb. 10). Die verfügbaren biomechanische Studien sind meist In-vitro-Untersuchungen und mit erwachsenen humanen Präparaten oder synthetischen Knochen eines Erwachsenen durchgeführt worden [10,11].

Eine sekundäre Dislokation ist im Kindealter nach 2 Wochen nahezu ausgeschlossen, und durch eine zusätzliche Gipsruhigstellung ist die Osteosynthese zusätzlich primär geschützt. Da immer 2 Verfahren in Kombination angewendet werden, ist die In-vitro-Studien zur Biomechanik nur bedingt verwertbar.

Beim Einbringen der K-Drähte ist zu beachten, dass die korrekte Lage intraoperativ im ulnoradialen Strahlengang zu prüfen ist So wird ein Rotationsfehler am ehesten sichtbar (Abb.5). Das Un- terlassen mehrfacher frustraner Bohrversuche ist wichtig, sodass keine Instabilität (durch eine Trümmerzone) und damit sekundäre Dislokation durch Ausbrechen des Drahts drohen (Abb. 11, 12). Der ulnare Draht wird von ventral nach dorsal eingebracht und der radiale Draht von dorsal nach ventral. Die Epikondylen können meist getastet oder durch eine Miniinzision tastbar gemacht werden, sodass die Drahteinbringung besser gelingt (Abb. 10).

Im Fall von nichttastbaren Kondylen kann vor einer Inzision initial im seitlichen Strahlengang die Mitte der Kondyle als Eintrittspunkt markiert werden (Punkt mit Pinzette). Die K-Drähte sollten sich oberhalb der Fraktur kreuzen. 

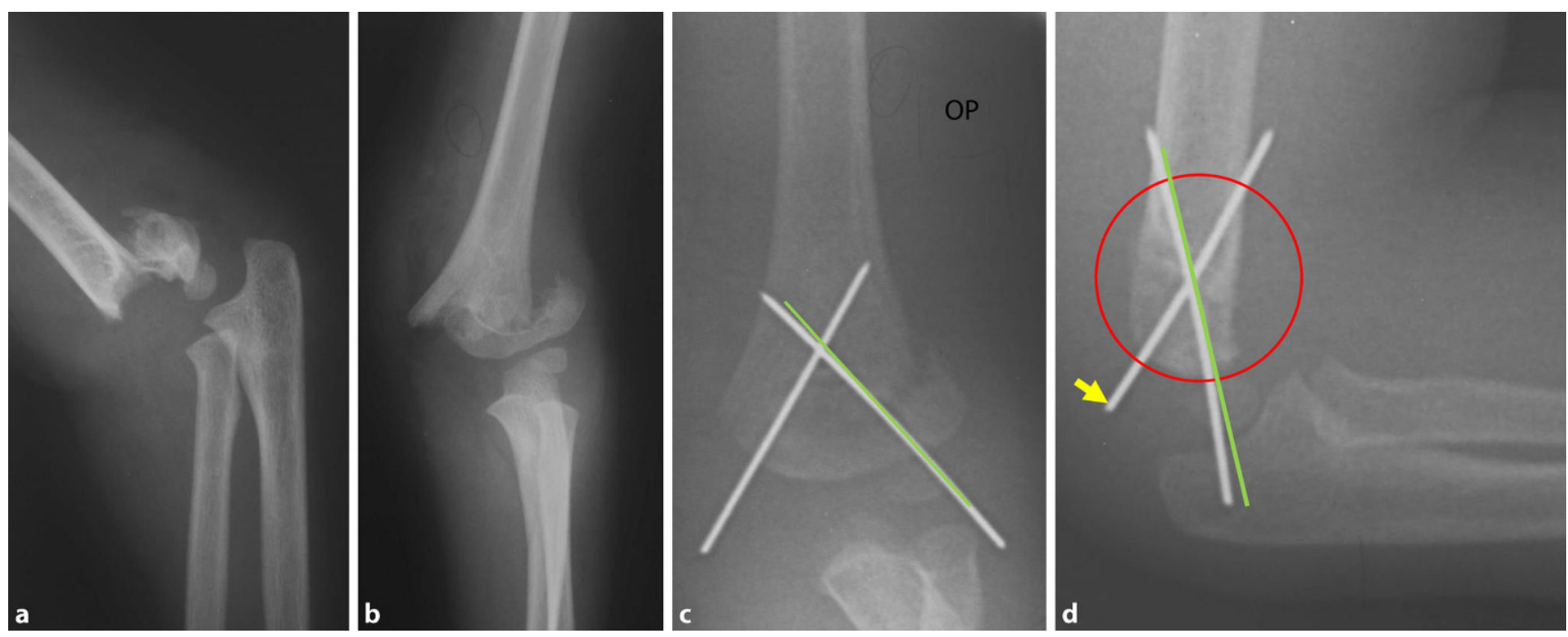

Abb. $13 \Delta$ a, b Typ-IV-Fraktur nach von Laer/AO. c, d Kirschner-Draht-Fixation. Ulnarer und radialer Draht wurden nicht korrekt eingebracht. Der ulnare Drahts ist zu weit dorsal platziert, mit der Gefahr der Verletzung des N. ulnaris. Der radiale Draht hätte ebenfalls in der seitlichen Ebene mehr in der Mitte eingebracht werden müssen

$\checkmark$ Merke

Korrektes Einbringen der K-Drähte:

- keine Kreuzung der K-Drähte im Frakturbereich,

- in der seitlichen Ebene möglichst mittig eingebrachte Drähte,

- „landmarks“ beachten, um keinen N.-ulnaris-Schaden zu produzieren,

- mehrfaches Bohren vermeiden (Abb. 13).

Elastisch stabile intramedulläre Nagelung. Die ESIN wird meist in französisch sprachigen Ländern angewendet und wurde von der Gruppe aus Nancy unter Prevot inauguriert [12]. Vorteil dieser Methode ist, dass die Lage der Schienen immer beurteilt und eine Fehlplatzierung schwer übersehen werden kann. Nachteile sind, dass während der Operation ein Assistent benötigt wird, die Materialentfernung nur im OP stattfinden kann und die Operationsdauer gegenüber einer unkomplizierten K-Draht-Versorgung verlängert ist. Des Weiteren ist die ESIN für die Versorgung sehr distaler Frakturen weniger geeignet.

Osteosynthesematerial. Es existiert nur eine Arbeit, in der resorbierbare Polymere verwendet wurden [13]. Für die Nutzung von resorbierbaren Materialien besteht ein großes Potenzial, und diese stellen einen weiteren Schritt dar, die optimale Therapie von Kindern zu gewährleisten.

\section{Zeitpunkt der operativen Versorgung}

In vielen Kliniken stellt die suprakondyläre Oberarmfraktur eine Notfalloperation dar. Dies gilt uneingeschränkt für Patienten mit Gefäß-Nerven-Läsionen.

Zwei Arbeiten aus dem Jahr 2018 erschienen in The Bone \& Joint Journal und Journal of Pediatric Orthopedic weisen darauf hin, dass die verzögerte Versorgung keine schlechteren Ergebnisse aufweist - natürlich unter Einbeziehung einiger Limitationen und auch der Tatsache, dass all diese Studien nicht prospektiv und/oder in genügend großen Populationen durchgeführt wurden. Es wird hervorgehoben, dass das Ausmaß der Expertise des Behandlers tagsüber deutlich besser ist [14, 15], was am Ende einen Vorteil für den Patient darstellt. Die Operation sollte am der Indikationsstellung folgenden Tag als Erste geplant werden.

\section{Gefäß-Nerven-Läsion}

Hier erscheint es wichtig, dass man grob orientierend zumindest die Motorik des N. medianus, ulnaris und radialis betreffend die Kinder einer solchen Untersuchung unterzieht. Zwei Arbeiten aus dem Jahr 2017 weisen darauf hin, dass die exakte primäre Untersuchung, die nur grob orientierend, meist die Motorik umfassend, und daher einfach durchzuführen ist, immer möglich sein sollte - spätestens aber nach der Operation erfolgen muss ([7, 16]; Abb. 14).

$>$ Merke

Von Komplikationen des initialen Frakturgeschehens ist am häufigsten der N. medianus oder die A. brachialis betroffen, gefolgt vom $\mathrm{N}$. radialis und nur selten des $\mathrm{N}$. ulnaris.

Die in der Literatur angegebene Rate der Gefäß-Nerven-Läsionen schwankt zentrenabhängig sowie bezogen auf das jeweilige Studiendesign zwischen $8 \%$ und $17 \%$; in manchen Publikationen ist diese Angabe noch höher [7, 16]. Beide zitierten Arbeiten weisen darauf hin, dass auch sekundäre Interventionen - den Nerv betreffend - gute Erfolgschancen zur Restitutio ad integrum aufweisen. Die operative Versorgung bei Verletzungen der A. brachialis ist eine Notfallindikation; die des N. medianus kann zeitverzögert erfolgen.

Um einen Gefäßschaden nicht zu übersehen, ist nach der Reposition zunächst die Perfusion zu prüfen. Hierzu sollte intraoperativ eine Duplexsonographie durchgeführt werden. Stellt sich eine Verletzung des Gefäßes dar, und kann die Perfusion durch Auflegen von warmen Tüchern nicht wiederhergestellt werden, ist die ope- 


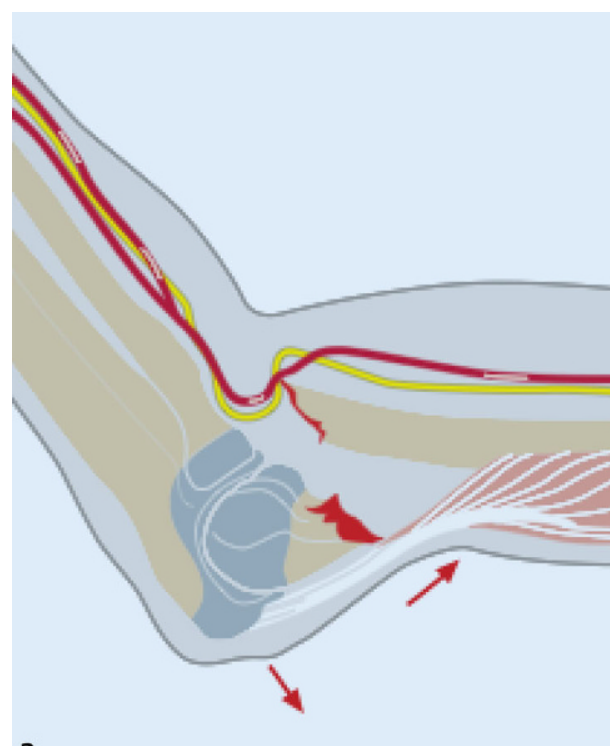

a
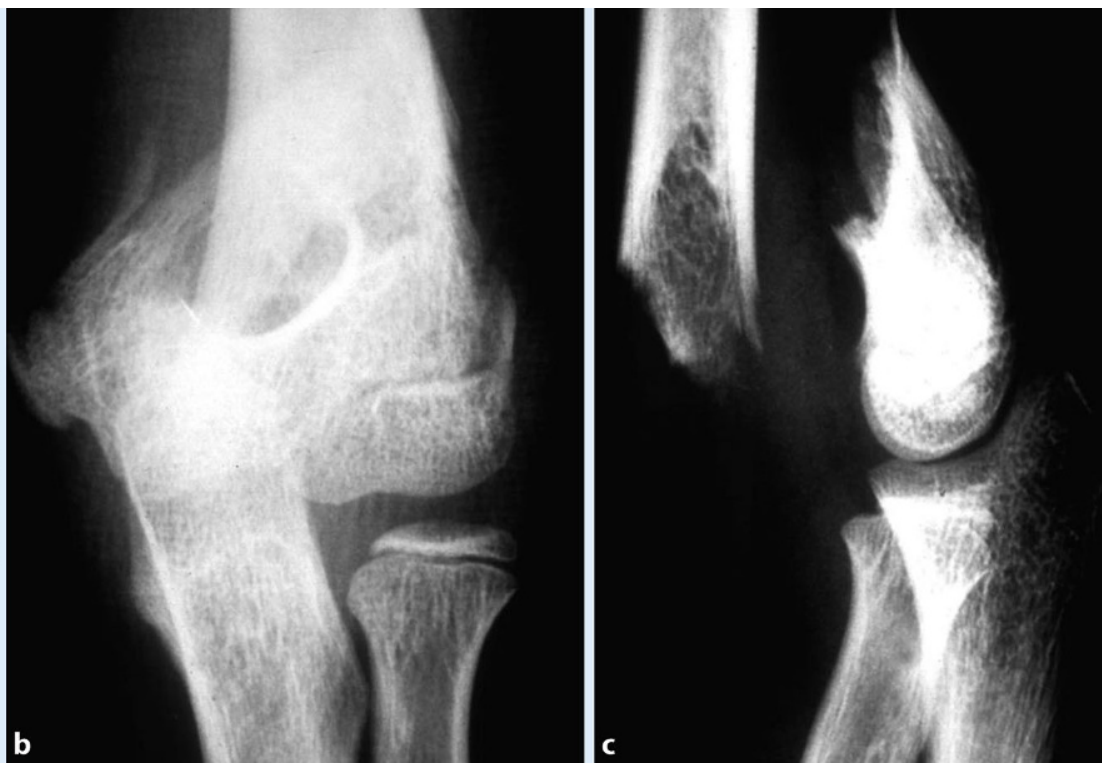

Abb. $14 \Delta$ a Schemazeichnung der Verletzung der A. brachialis bzw. des N. medianus. b, c Radiologische Bilder einer vollständig dislozierten Fraktur mit Gefahr der Verletzung des N. medianus und der A. brachialis. (Aus [4]. Dieser Inhalt ist nicht Teil der Open-Access-Lizenz)

rative Revision angezeigt. Hier weist der vordere Zugang zum Ellenbogen Vorteile auf [17].

\section{$>$ Cave}

Eine N.-ulnaris-Läsion stellt meist eine Komplikation der Operation dar.

Bei Läsionen des N. ulnaris werden in der Literatur das Zuwarten, aber auch die Revision empfohlen [18]. Eigene Erfahrungen in Österreich zeigen, dass nach Entfernung der Drähte mit einer guten Regeneration des Nervs gerechnet und daher insbesondere im Kindealter zugewartet werden kann. Dies gilt insbesondere, da der Nerv nicht durchtrennt, sondern eingeengt oder durch den Draht fixiert ist und so verletzt wurde. Die Autoren des vorliegenden Beitrags verzichten eher auf die primäre Revision des Nervs im Gegensatz zu den deutschen Kollegen, die gemäß einer Analyse aus dem Jahr 2019 der Revision den Vorzug geben [18].

$$
\text { - Wichtig }
$$

Bei Schäden des N. ulnaris sind das frühzeitige Erkennen und die Aufklärung der Eltern über den absehbaren längeren Heilungsverlauf wichtig.

Die Regenerationszeit beträgt zwischen 3 und 9 Monate; die Dauer hängt von der Heilung des Nervs, der Art des Schadens und vom Patientenalter ab.

$$
\text { - Merke }
$$

Das Hoffmann-Tinel-Zeichen kann zur Erfolgsbeurteilung eines sich erholenden Nervs und zu dessen Beurteilung der Regeneration herangezogen werden.

Das Hoffman-Tinel-Zeichen wird beschrieben zur Überprüfung von chirurgischen Nervennähten, ist aber für Kinder in der klinischen Praxis bei Nervenschäden, insbesondere des N. ulnaris, sehr gut geeignet, um den Regenerationsverlauf in der Ambu- lanz anzuzeigen: Bei einer unkomplizierten Reinnervation kann ein Wachstum des Axons von etwa 1-5 mm/Tag erwartet werden. Die Stelle im Verlauf des Nervs, an der das Zeichen positiv wird, zeigt dessen aktuelle Position. Sie sollte sich im Idealfall also entlang der Nervenstraße allmählich in Richtung Erfolgsorgan bewegen. Ein Kind kann sehr genau sagen, wo es entweder elektrische Potenziale (wie beim Musikantenknochen) bemerkt oder das Gefühl einfach anders ist.

Auch bei korrektem Einbringen der K-Drähte kommt es immer wieder zu einer Einengung des N. ulnaris. Manche Autoren postulieren das einheitliche offene Vorgehen zur Versorgung, was aus der Literatur nicht nachvollzogen werden kann. Klinikintern sollten klare Richtlinien für ein offenes Vorgehen definiert sein. Hierzu gehört auch der Schutz des N. ulnaris durch eine Miniinzision, über die der Epicondylus ulnaris getastet werden kann. Der Draht wird dann ventral hiervon eingebracht $[1,3]$.

\section{Nachbehandlung}

\section{Materialentfernung}

Die Fraktur ist unabhängig von der gewählten Therapie nach 3 bis 4 Wochen konsolidiert [1, 2, 3]. Die Implantate können ab diesem Zeitpunkt entfernt werden. Herausstehende Drähte lassen sich beim durch den Unfallchirurg/Kinderarzt oder Anästhesisten analgosedierten Kind einfach entfernen und bedürfen keiner Kapazität im OP. Alle anderen Osteosyntheseverfahren werden zumeist im OP entfernt.

- Merke

Die Metallentfernung erfolgt spätestens nach 4 bis 5 Wochen.

\section{Postoperatives Schmerzregime}

Das postoperative Schmerzregime nach suprakondylärer Humerusfraktur umfasst die Gabe von Schmerzmitteln für insgesamt 
3 Tage als feste Medikation und anschließend nach Bedarf. In einer Studie (Januar 2019) mit 81 Patienten, veröffentlicht in The Journal of Bone \& Joint Surgery konnte gezeigt werden, dass die betreffenden Kinder meist nach 3 Tagen schmerzfrei sind und fast keine Schmerzmittel mehr benötigen $[19,20]$. Einer kontinuierlichen Therapie wird der Vorzug gegeben, um Schmerzspitzen zu vermeiden.

Die Studie und eigene klinische Erfahrung weisen darauf hin, dass „outliners", die einen besonders hohen Schmerzmittelbedarf haben, ein Indiz dafür sein können, dass sich hinter einem hohen Schmerzmittelbedarf Komplikationen wie ein Kompartmentsyndrom oder eine Ischämie verstecken können. Die Autoren empfehlen, diese Kinder besonders kritisch zu überwachen.

\section{> Merke}

Insgesamt sollte eine kontinuierliche Schmerztherapie über 3 bis 5 Tage verordnet werden, da Eltern ihren Kindern aus unbegründeten Ängsten oftmals gar keine Schmerzmittel verabreichen.

\section{Resümee}

Die suprakondyläre Oberarmfraktur im Kindesalter ist und bleibt eine Herausforderung, zu deren Versorgung sich immer neue oder sich ändernde Aspekte ergeben. Dies hat die deutschsprachigen Gesellschaften bewogen, Diagnose- und Versorgungsstandards zu definieren, die laufend überarbeitet und angepasst werden [21]. Wichtige Fragestellungen können nur im Rahmen großer multizentrischer prospektiver Studien ausreichend untersucht werden. Auch die Ausbildung und das Training der versorgenden Ärzte müssen sich für die zukünftige Generation ändern, da dem Modell „see and learn" nicht mehr gefolgt werden kann [22]. Simulationen können die operative Situation detailgetreu nachstellen und damit dazu beitragen, die einzelnen Operationsschritte frakturabhängig zu trainieren, aber auch potenzielle Fehler zu analysieren.

\section{Fazit für die Praxis}

- Die suprakondyläre Oberarmfraktur kann zuverlässig durch die Klassifikationen nach von Laer/AO eingeteilt werden. Ein Therapiealgorithmus lässt sich ableiten.

- Typ-I-Frakturen sind konservativ zu behandeln. Typ-II-Frakturen (auBer Flexionsfrakturen) können durch Redression konservativ behandelt werden; Typ-III- und Typ-IV-Frakturen müssen operativ versorgt werden.

- Fehlstellungen können sich nur in der Sagittaleben bis zum 6. Lebensjahr remodellieren; ein Cubitus varus bzw. valgus korrigiert sich nicht im Laufe des Wachstums.

- Die zeitnahe Versorgung mithilfe von Kirschner-Drähten, Fixateur externe oder der elastisch stabilen intramedullären Nagelung (ESIN) sollte angestrebt werden.

- Die Problematik der Fraktur besteht im sekundären Abkippen oder dem Repositionsverlust. Dies ist auf die Abnahme der Kontaktfläche bei Rotationsfehlstellung zurückzuführen, wobei der Rotationsfehler am ehesten in der ulnoradialen Röntgenbildeinstellung diagnostiziert werden kann.
- Am häufigsten treten zusätzlich Schäden der A. brachialis und des N. medianus auf; der N. ulnaris wird meist intraoperativ geschädigt.

- Die Fraktur ist nach 3 bis 4 Wochen konsolidiert; anschließend kann die Implantatentfernung erfolgen. Eine postoperative Schmerzmittelgabe ist für 3 bis 5 Tage fest zu verordnen.

\section{Korrespondenzadresse}

\section{Assoc. Prof. Dr. Annelie-Martina Weinberg}

Univ. Klinik für Orthopädie und Orthopädische Chirurgie, Medizinische Universität Graz

Auenbruggerplatz 5, $8036 \mathrm{Graz}$, Österreich

anneliemartina.weinberg@medunigraz.at

Funding. Open access funding provided by Medical University of Graz.

\section{Einhaltung ethischer Richtlinien}

Interessenkonflikt. Gemäß den Richtlinien des Springer Medizin Verlags werden Autoren und Wissenschaftliche Leitung im Rahmen der Manuskripterstellung und Manuskriptfreigabe aufgefordert, eine vollständige Erklärung zu ihren finanziellen und nichtfinanziellen Interessen abzugeben.

Autoren. A.-M. Weinberg: Finanzielle Interessen: bezahlter Berater/interner Schulungsreferent/Gehaltsempfänger o. Ä.: Fa. Bioretec Oy, Finnland. - Patente, Geschäftsanteile, Aktien o. Ä. an einer im Medizinbereich aktiven Firma: Aktien der Fa. Bioretec. - Nichtfinanzielle Interessen: Ordination stundenweise, Berater bei W\&M, Leiter der Study Group Trauma EPOS, AO member und faculty, ÖGU-Kindertraumatologie. B. Frei: Finanzielle Interessen: B. Frei gibt an, dass kein finanzieller Interessenkonflikt besteht. - Nichtfinanzielle Interessen: Universitäts-Kinderspital beider Basel (UKBB): Januar 2014 bis Juni 2017 Assistenzarzt, Juli 2017 bis August 2019 stv. Oberarzt, September 2019 bis August 2020 Assistenzarzt, ab September 2020 stv. Oberarzt. P. Holweg: Finanzielle Interessen: P. Holweg gibt an, dass kein finanzieller Interessenkonflikt besteht. - Nichtfinanzielle Interessen: angestellter Orthopäde und Unfallchirurg an der Medizinischen Universität Graz.

Wissenschaftliche Leitung. Die vollständige Erklärung zum Interessenkonflikt der Wissenschaftlichen Leitung finden Sie am Kurs der zertifizierten Fortbildung auf www. springermedizin.de/cme.

Der Verlag erklärt, dass für die Publikation dieser CME-Fortbildung keine Sponsorengelder an den Verlag fließen.

Für diesen Beitrag wurden von den Autoren keine Studien an Menschen oder Tieren durchgeführt. Für die aufgeführten Studien gelten die jeweils dort angegebenen ethischen Richtlinien. Für Bildmaterial oder anderweitige Angaben innerhalb des Manuskripts, über die Patienten zu identifizieren sind, liegt von ihnen und/oder ihren gesetzlichen Vertretern eine schriftliche Einwilligung vor.

Open Access. Dieser Artikel wird unter der Creative Commons Namensnennung 4.0 International Lizenz veröffentlicht, welche die Nutzung, Vervielfältigung, Bearbeitung, Verbreitung und Wiedergabe in jeglichem Medium und Format erlaubt, sofern Sie den/die ursprünglichen Autor(en) und die Quelle ordnungsgemäßnennen, einen Link zur Creative Commons Lizenz beifügen und angeben, ob Änderungen vorgenommen wurden.

Die in diesem Artikel enthaltenen Bilder und sonstiges Drittmaterial unterliegen ebenfalls der genannten Creative Commons Lizenz, sofern sich aus der Abbildungslegende nichts anderes ergibt. Sofern das betreffende Material nicht unter der genannten Creative Commons Lizenz steht und die betreffende Handlung nicht nach gesetzlichen Vorschriften erlaubt ist, ist für die oben aufgeführten Weiterverwendungen des Materials die Einwilligung des jeweiligen Rechteinhabers einzuholen.

Weitere Details zur Lizenz entnehmen Sie bitte der Lizenzinformation auf http:// creativecommons.org/licenses/by/4.0/deed.de. 


\section{Literatur}

1. Wessels L (2016) Ellenbogen. In: Marzi I (Hrsg) Kindertraumatologie. Springer, Berlin Heidelberg, S150-167

2. Rockwood CA, Wilkins KE, King RE (1984) Supracondylarfractures. In:RockwoodCA (Hrsg) Fractures in children, 3. Aufl. Lippincott, Philadelphia, S376-428

3. Weinberg A, Castellani C (2002) Distaler Oberarm - supracondyläre Oberarmfraktur. In: Weinberg T (Hrsg) Unfallchirurgie im Kindesalter. Teil 1. Springer, Heidelberg, S217-249

4. Weinberg AM, Castellani C (2006) Distaler Oberarm - suprakondyläre Oberarmfraktur. In: Weinberg AM, Tscherne $\mathrm{H}$ (Hrsg) Tscherne Unfallchirurgie Band 1: Unfallchirurgie im Kindesalter (Allgemeiner Teil Kopf Obere Extremität Wirbelsäule), Springer, Berlin

5. Weinberg AM, Marzi I, Günter SM, Wessel L, Riedel J, von Laer L (2002) Die suprakondyläre Oberarmfraktur im Kindesalter - eine Effizienzstudie. Teil 1 Epidemiologie, Effektivitätsprüfung und Klassifikation. Unfallchirurg 105:208-216

6. Ernat J, Ho C, Wimberly RL, Jo C, Riccio Al (2017) Fracture classification does not predict functional outcomes in supracondylar humerus fractures: a prospective study. Pediatr Orthop 37(4):27776052. https://doi.org/10.1097/BPO. 0000000000000889

7. v. Laer L, Kraus R (2009) Komplikationen bei der Behandlung von Verletzungen im Wachstumsalter.In:Wirth CJ, MutschlerW, BischoffH-P, Plüschmann H, Neu J (Hrsg) Komplikationen in Orthopädie und Unfallchirurgie. Thieme, Stuttgart, S514-552

8. Muccioli C et al (2017) Outcomes of Gartland type III supracondylar fractures treated using Blount's method. Orthop Traumatol Surg Res. https://doi.org/10. 1016/j.otsr.2017.06.011

9. Li NY, Bruce WJ, Joyce C, Decker NM, Cappello T (2018) Obesity's influence on operative management of pediatric supracondylar humerus fractures. J Pediatr Orthop 38(3): e118-e121

10. Kamara A, Ji X, Liu T, Zhan Y, Li J, Wang E (2019) A comparative biomechanical study on different fixation techniques in the management of transverse metaphysealdiaphyseal junction fractures of the distal humerus in children. Int Orthop 43:411-416

11. Wallace M, Johnson DB, Pierce W, lobst C, Riccio A, Wimberly RL (2019) Biomechanical assessment of torsional stiffness in a supracondylar humerus fracture model.J Pediatr Orthop 39(3):e210-e215

12. Prevot J, Lascombes $P$, Metizeau JP, Blanquet $D(1990)$ Supracondylar fractures of the humerus in children - Treatment by downward pinning. Rev Chir Orthop 76:191-197

13. Li J et al (2017) Surgical management of delayed irreducible Gartland III supracondylar fractures in children: open reduction and internal fixation versus external fixation.JShoulder Elbow Surg.https://doi.org/10.1016/j.jse.2016.10.006

14. Farrow $L$ et al (2018) Early versus delayed surgery for paediatric supracondylar humeral fractures in the absence of vascular compromise. Bone Joint J. https://doi. org/10.1302/0301-620X.100B12.BJJ-2018-0982.R1

15. Tuomilehto $\mathrm{Netal}$ (2018) Postponing surgery of paediatric supracondylar humerus fractures to office hours increases consultant attendance in operations and saves operative room time. JChild Orthop. https://doi.org/10.1302/1863-2548.12. 170144

16. Leiblein M et al (2017) Neurovascular complications after supracondylar humerus fractures in children. https://doi.org/10.1016/j.tcr.2017.01.013

17. Su Y, Nan G (2019) Evaluation of a better approach for open reduction of severe Gartland type III supracondylar humeral fracture. J Invest Surg. https://doi.org/10. 1080/08941939.2019.1649766

18. Kraus R, Schmittenbecher $P$ (2019) Was tun bei postoperativem Ulnarisschaden nach Kirschner-Draht-Osteosynthese der suprakondylären Humerusfraktur im Kindesalter? Unfallchirurg. https://doi.org/10.1007/s00113-019-0629-5

19. Tuomilehto $\mathrm{N}$ et al (2018) Postponing surgery of paediatric supracondylarhumerus fractures to office hours increases consultant attendance in operations and saves operative room time. J Child Orthop. https://doi.org/10.1302/1863-2548.12. 170144

20. Nelson SE, Adams AJ, Buczek MJ, Anthony CA, Shah AS (2019) Postoperative pain and opioid use in children with supracondylar humeral fractures: balancing analgesia and opioid stewardship. J Bone Joint Surg Am 101(2):119-126. https:// doi.org/10.2106/JBJS.18.00657.

21. AMWFLeitlinien:https://www.awmf.org/leitlinien/detail/ll/012-014.html

22. Holt JB et al (2017) Emerging U.S. National Trends in the Treatment of Pediatric Supracondylar Humeral Fractures. Bone Joint Surg. https://doi.org/10.2106/JBJS. 16.01209 
Zu den Kursen dieser Zeitschrift: Scannen Sie den QR-Code

oder gehen Sie auf www.springermedizin.de/kurse-der-unfallchirurg

? Welches ist die gebräuchlichste Einteilung der suprakondylären Oberarmfraktur im deutschsprachigen Raum?

O Gartland-Klassifikation

- Felsenreich-Klassifikation

O Lubinus-Klassifikation

○ Von-Laer/AO-Klassifikation

○ Baumann-Klassifikation

Ein 5-jähriger Junge erleidet aufgrund eines Sturzes eine suprakondyläre Oberarmfraktur. Welchen der folgenden Typen der Von-Laer-/AOKlassifikation würden Sie anhand des ersichtlichen a.-p.- und seitlichen Röntgenbilds diagnostizieren?
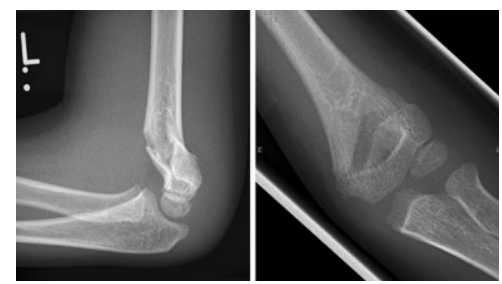

○ Typ I

○ Typ II Flexionsfehlstellung

O Typ II Extensionsfehlstellung

O Typ III

O Typ IV
? Ein 7-jähriges Mädchen verunfallt auf dem Trampolin und zieht sich eine suprakondyläre Oberarmfraktur des Typs II (Flexionsfraktur) nach von Laer zu. Wie würden Sie vorgehen?

O Oberarmgips, da sich die Fehlstellung in diesem Alter remodelliert

○ Anlage einer Blount-Schlinge („Collar-andcuff"-Verband) für 6 Wochen, keine weiteren Kontrollen

○ Anlage einer Blount-Schlinge („COllar-andcuff"-Verband) und Kontrolle der Redression nach 3 bis 5 Tagen

O Es sollte eine elastische Wickelung mit frühfunktioneller Nachbehandlung erfolgen

O Im OP eine Reposition durchführen, wenn die Fraktur stabil ist, weiter konservativ vorgehen, sonst operativ stabilisieren

Was versteht man unter der RogersHilfslinie?

O Der Winkel zwischen Humeruslängsachse und Epiphysenfugenachse soll im seitlichen Strahlengang zwischen 30 und $40^{\circ}$ betragen.

O Die Hilfslinie wird zur Abschätzung der Achse des Ellbogengelenks genutzt, insbesondere wenn der Arm nicht gestreckt und supiniert werden kann.

○ Diese beschreibt im seitlichen Strahlengang eine Tangentiale entlang der vorderen Humeruskortikalis, deren Verlängerung im Normalfall das Capitulum humeri am
Übergang vom mittleren zum hinteren Drittel schneidet.

O Wird gemessen vom proximale Humerus nach distal als Mittellinie bezogen auf einen $90^{\circ}$ Winkel im Ellenbogengelenk.

○ Sie bezieht sich auf die Stellung des Radiusköpfchens zur proximalen Ulna.

? Ein 11-jähriger Junge stürzt von der Schaukel auf den Arm. Das konventionelle Röntgenbild des Ellenbogengelenks zeigt eine suprakondyläre Humerusfraktur des Typs IV nach von Laer. Welche Behandlung würden Sie am ehesten anstreben?

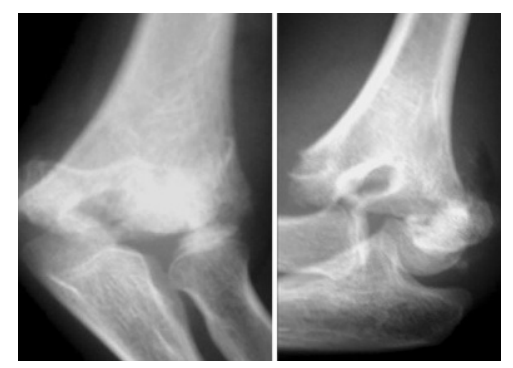

O Reposition und konservative Weiterbehandlung

O Reposition und Platte

O Reposition und Schrauben

○ Reposition und primäre Fixateur-externeAnlage

O Reposition und gekreuzte Kirschner-DrahtOsteosynthese

\section{Informationen zur zertifizierten Fortbildung}

Diese Fortbildung wurde von der Ärztekammer Nordrhein für das „Fortbildungszertifikat der Ärztekammer" gemäß $§ 5$ ihrer Fortbildungsordnung mit 3 Punkten (Kategorie D) anerkannt und ist damit auch für andere Ärztekammern anerkennungsfähig.
Anerkennung in Österreich: Für das Diplom-Fortbildungs-Programm (DFP) werden die von deutschen Landesärztekammern anerkannten Fortbildungspunkte aufgrund der Gleichwertigkeit im gleichen Umfang als DFP-Punkte anerkannt (§ 14, Abschnitt 1, Verordnung über ärztliche Fortbildung, Österreichische Ärztekammer (ÖÄK) 2013).
Hinweise zur Teilnahme:

- Die Teilnahme an dem zertifizierten Kurs ist nur online auf www.springermedizin.de/cme möglich.

- Der Teilnahmezeitraum beträgt 12 Monate. Den Teilnahmeschluss finden Sie online beim Kurs.

- Die Fragen und ihre zugehörigen Antwortmöglichkeiten werden online in zufälliger Reihenfolge zusammengestellt.
- Pro Frage ist jeweils nur eine Antwort zutreffend.

- Für eine erfolgreiche Teilnahme müssen 70\% der Fragen richtig beantwortet werden.

- Teilnehmen können Abonnenten dieser Fachzeitschrift und e.Med-Abonnenten.

- Abonnenten von „Der Orthopäde“ oder „Der Unfallchirurg" können kostenlos an CME-Kursen beider Zeitschriften teilnehmen. 
?elche Komplikation nach Osteosynthese einer suprakondylären Humerusfraktur mithilfe gekreuzter KirschnerDrähte stellt am ehesten einen intraoperativen Schaden dar?

○ Nervus-radialis-Läsion

○ Nervus-medianus-Läsion

○ Nervus-ulnaris-Läsion

○ Arteria-brachialis-Läsion

○ Vena-mediana-cubiti-Läsion

? Wie kann man die Regeneration des N. ulnaris nach einer Läsion in der Ambulanz sehr einfach prüfen?

O Hoffmann-Tinel-Zeichen

O Trizeps-Sehnen-Reflex

○ Messung der Nervenleitgeschwindigkeit

O Phalen-Zeichen

O Kapandji-Score
? Welche Ebene korrigiert sich im Wachstum nach suprakondylärer Oberarmfraktur im Kindesalter nicht?

O Rekurvationsebene

O Antekurvationsebene

O Sagittalebene

O Frontalebene

O Humeruskopf-Ellenbogengelenk-Ebene

?elches Osteosynthesematerial kommt am häufigsten bei der Versorgung der suprakondylären Oberarmfraktur zum Einsatz?

O Kirschner-Drähte

O Platten

O Resorbierbare Pins

○ Elastisch stabile intramedulläre Nagelung (ESIN)

O Fixateur externe
? Eine 8-jährige Patientin weist nach einer suprakondylären Humerusfraktur eine Überstreckung und ein Beugedefizit im Ellenbogengelenk auf, keinen Cubitus varus. Um welche Achsenfehlstellung handelt es sich?

O Flexionsfehlstellung

O Rotationsfehlstellung

- Fehlstellung in der Frontalebene

O Extensionsfehlstellung

O Fehlstellung als Folge einer Wachstumsstörung

\section{Doppelt punkten!}

Schon gewusst: Als Abonnent von Der Unfallchirurg nehmen Sie kostenlos an Fortbildungen von Der Orthopäde teil!

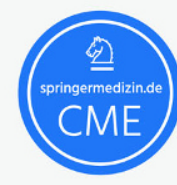

Aktuelle Fortbildungen in Der Orthopäde

- Chronische patellofemorale Instabilität

- Differenzialdiagnostik akuter Rückenschmerz

- Osteonekrose großer Gelenke

- Atraumatische Frakturen der Wirbelsäule

- Omarthrose

- Doping im Spitzen- und Breitensport

\section{Springer Medizin}

www.SpringerMedizin.de/cme

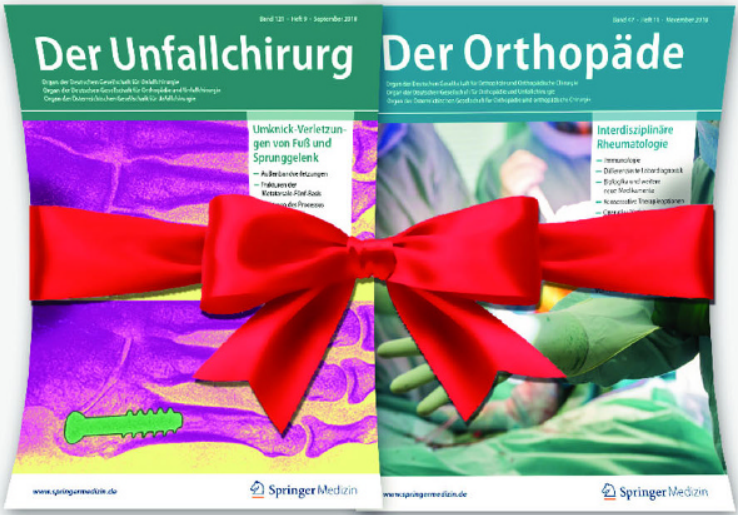

\section{Gratis}

So nehmen Sie teil: teilnehmen!

1. Melden Sie sich auf SpringerMedizin.de/cme

mit Ihren Zugangsdaten an.

2. Es werden Ihnen automatisch die Fortbildungen beider Zeitschriften angezeigt. Um den Service nutzen zu können, muss Ihre Abonummer unter „Meine Daten“ hinterlegt sein.

3. Gewünschte Fortbildung auswählen, „Kurs starten“ und CME-Punkte sammeln. 